ESAIM: PROCEEDINGS, April 2012, Vol. 36, p. 126-158

Danièle Fournier-Prunaret, Laura Gardini, \& Ludwig Reich, Editors

\title{
UNSTABLE ORBITS AND MILNOR ATTRACTORS IN THE DISCONTINUOUS FLAT TOP TENT MAP
}

\author{
Viktor Avrutin $^{1}$, Ben Futter ${ }^{2}$, Laura Gardini ${ }^{3}$ And Michael Schanz ${ }^{4}$
}

\begin{abstract}
In this work we consider the discontinuous flat top tent map which represents an example for discontinuous piecewise-smooth maps, whereby the system function is constant on some interval. Such maps show several characteristics caused by this constant value which are still insufficiently investigated. In this work we demonstrate that in the discontinuous flat top tent map every unstable periodic orbit may become a Milnor attractor. Moreover, it turns out that there exists a strong connection between stable and unstable orbits and that the appearance of a single unstable orbit may cause an infinite number of stable orbits to appear. Based on this connection we provide a more precise explanation of the recently discovered self-similar bifurcation scenario occurring in the discontinuous flat top tent map denoted as the nested period incrementing scenario.
\end{abstract}

AMS (2000) subject classification. 37E05, 37E15, 37G35.

Keywords. piecewise-smooth maps; discontinuous flat top tent map; maps with a horizontal part; nested period incrementing; Milnor attractors; U-sequence.

Résumé. Dans ce travail nous considérons l'application discontinue de tente à haut plat qui représente un exemple d'application régulière par morceaux discontinue, où la fonction du système est constante sur un intervalle. De telles applications montrent plusieurs aspects causés par cette valeur constante qui ne sont toujours pas suffisamment compris. Dans ce travail nous démontrons que pour l'applicatiion discontinue de tente à haut plat toutes les orbites périodiques instables peuvent devenir un attracteur de Milnor. De plus, il apparaît qu'il y a un forte connexion entre les orbites stables et instables et que l'apparition d'une seule orbite instable peut provoquer l'apparition d'un nombre infini d'orbites stables. Sur la base de cette connexion nous proposons une explication précisée du scénario de bifurcation auto-similaire découvert récemment pour l'applicatiion discontinue de tente à haut plat, le scénario d'incrément de période nichée.

Mots clefs : applications régulières par morceaux ; application discontinue de tente à haut plat ; applications avec une partie horizontale ; incrément de période nichée ; attracteur de Milnor ; $U$-suite.

\section{INTRODUCTION}

When dealing with a bifurcation scenario, it is natural to ask which periodic orbits are involved. The first and not very precise answer may be given by the periods of the orbits. So, for example, the period-doubling scenario starting with some orbit with the period $p_{0}$ will be usually described as a sequence of orbits with the

${ }^{1}$ Institute of Parallel and Distributed Systems, University of Stuttgart, Germany Viktor.Avrutin@ipvs.uni-stuttgart.de.

2 Institute of Parallel and Distributed Systems, University of Stuttgart, Germany Ben.Futter@ipvs.uni-stuttgart.de.

3 Department of Economics, Society and Politics, University of Urbino, Italy, Laura.Gardini@uniurb.it.

${ }^{4}$ Institute of Parallel and Distributed Systems, University of Stuttgart, Germany Michael.Schanz@ipvs.uni-stuttgart.de.

(C) EDP Sciences, SMAI 2012 
periods $p_{n}=p_{0} \cdot 2^{n}$ with $n \geq 0$. For the period doubling scenario this kind of description is usually sufficient, but in general it is not, since a scenario may also include several orbits with the same period. In such cases a more precise description of the bifurcation scenario is needed, which can be provided for example by the symbolic sequences of the periodic orbits forming the scenario. In this case the question arises, whether all symbolic sequences in a particular bifurcation scenario - or at least some of them - can be written explicitly, in a closed form. By contrast to the periods, the answer is - typically not (already for the period doubling we can provide only a rule how to create the sequences up to a given period, see $\S 2.1$ for details). Similarly, for the self-similar period adding structure where between the existing regions of two orbits with some periods $p$ and $p^{\prime}$ the existing region of the orbit with the period $p+p^{\prime}$ is located, we can provide a complete symbolic description by the Farey-tree-like symbolic sequences adding scheme. Up to now, the period incrementing scenario (for which the periods of the involved orbits form the arithmetic series $p_{n}=p_{0}+n \Delta p$ with some constant increment $\Delta p)$ seems to be the only bifurcation scenario where all the sequences can be written explicitly ${ }^{1}$.

The theory of piecewise-smooth dynamical systems represents nowadays a well established and rapidly growing domain, accepted by the scientific community both from the theoretical and the practical point of view. However, there exists a class of piecewise-smooth maps which was barely investigated until now, namely the maps defined on many partitions, whereby on one of the partitions the system function is constant. In the following we denote such systems as piecewise-smooth systems with a horizontal part. Several practical applications of such models are known, as for example a simple limiter control of unimodal maps [5, 14, 15, 18, 19], which is applicable for chaos control or the control of cardiac arrhythmia [7,8], as well as some electronic converters [11]. However, the theoretical basis regarding the bifurcation scenarios in such maps still has to be established.

In previous publications $[2,4]$ we explained the mechanism leading to a new bifurcation scenario, which we denoted as the nested period incrementing scenario. In fact, this scenario was recently observed experimentally (see [10]) but neither investigated nor explained in the cited work. For the investigation of this scenario we introduced in [2] a 1D map, which we denoted as the discontinuous flat top tent map and which represents a discontinuous variant of the well-known flat top tent map initially introduced in [12]. For the discontinuous flat top tent map we described the bifurcation structure in a $2 \mathrm{D}$ parameter space $(\alpha, \beta)$ (for details regarding the meaning of the parameters see $\S 1.1$ below), and then we faced the following problem. For small values of $\alpha$ one can easily specify all the orbits forming the bifurcation scenario which can be observed for varying $\beta$. In fact, this bifurcation scenario is given by two period incrementing cascades, and hence the symbolic description of every orbit is known. For large values of $\alpha$ we proved that for any $n$ a stable periodic orbit exists corresponding to every symbolic sequence of length $n$. However, the question arises whether it is possible to specify the orbits existing for values of $\alpha$ between these two limiting cases, that means for the values of $\alpha$ for which the bifurcation scenario for varying $\beta$ is more complicated than two incrementing cascades, but for which still not every sequence is possible.

Basically, this question can be answered positively. In [2] we presented an algorithmic approach which allows to decide whether a stable periodic orbit corresponding to a given symbolic sequence exists for a given value of $\alpha$. Therefore, we can generate the set of all symbolic sequences corresponding to stable periodic orbits up to a given period existing at any fixed value of $\alpha$. The approach is based on a complete binary tree of sequences, whereby at each step of the generation of this tree some analytic conditions must be verified in order to decide whether the next node of the tree exists or not. The obvious disadvantage of this approach is that the sequences are not grouped in families but every single sequence must be created in a separate step. This disadvantage is not crucial, since in the case of the Farey-tree-like symbolic sequences adding scheme, as well as in the case of the symbolic sequences corresponding to orbits forming a period-doubling cascade the situation is similar. However, there is a further and slightly more serious disadvantage of this approach. From the bifurcation diagrams of the scenario at a given value of $\alpha$ (see $\S 1.1)$, it becomes immediately clear that the stable periodic

\footnotetext{
${ }^{1}$ for example, when dealing with piecewise-smooth maps defined on two partitions, this scenario is frequently formed by families of so-called maximal periodic orbits, and the corresponding symbolic description is given in this case by families of symbolic sequences $\left\{\mathcal{L R}^{n} \mid n>0\right\}$ and $\left\{\mathcal{R} \mathcal{L}^{n} \mid n>0\right\}$, whereby the letters $\mathcal{L}$ and $\mathcal{R}$ refer to the points located on different sides of the boundary between the partitions (switching manifold).
} 
orbits forming this scenario are organized in some families. Clearly, the approach mentioned above can not provide any information about these families.

A more detailed investigation of the discontinuous flat top tent map demonstrates that this system shows an unexpected connection between stable and unstable periodic orbits. As we will see below, the existence of an unstable periodic orbit at a given value of $\alpha$ implies the existence of some infinite families of stable periodic orbits at this value of $\alpha$. This connection is based on some properties, which are specific not only for the discontinuous flat top tent map but in general for piecewise-smooth maps with a horizontal part. Therefore, the aim of this paper is to report these properties and to explain the connection between stable and unstable periodic orbits in the discontinuous flat top tent map, keeping in mind that other piecewise-smooth maps with a horizontal part may show the same or at least similar phenomena.

The paper is organized as follows. First, in $\S 1$ we present a brief overview summarizing the results already presented in the previous publications regarding the bifurcation structure formed by stable orbits. Then, in $\S 2$ we report the results regarding the connection between stable and unstable periodic orbits mentioned above. In $\S 2.1$ we describe an infinite sequence of unstable periodic orbits (similar to orbits forming a period doubling cascade in the logistic map and corresponding to the same symbolic sequences), converging to some parameter value $\alpha_{\infty}$. Then, in $\S \S 2.2$ and 2.3 we consider the first orbit in this sequence, namely an unstable fixed point, and explain, which stable periodic orbits appear at the same parameter value as this fixed point. Additionally, in $\S 2.4$ we describe some properties of the stable and unstable sets of this fixed point. Next, in $\S 2.5$ we consider the unstable period- 2 orbit and the general situation up to the parameter value $\alpha_{\infty}$. As a final result, we obtain an explicit symbolic description of all stable periodic orbits existing at any parameter value $\alpha<\alpha_{\infty}$. Furthermore, in $\S 2.6$ we demonstrate that the other unstable fixed point of the discontinuous flat top tent map (not considered before) shows some properties similar to all other unstable orbits already described and explain the differences. In $\S 3$ we discuss the situation for $\alpha>\alpha_{\infty}$ and explain why it seems to be not possible to derive and explicit symbolic description of all stable periodic orbits existing in this case. Finally, in $\S 4$ we summarize the obtained results and emphasize which of them are specific for the discontinuous flat top tent map and which of them have a more general meaning for other systems with a horizontal part.

\section{Overall Bifurcation Structure Formed by Stable Orbits}

\subsection{Definition of the map}

The map we are investigating in this work is defined by

$$
x_{n+1}=f\left(x_{n}\right), \quad f(x)= \begin{cases}f_{\mathcal{L}}(x)=2 \alpha x & \text { if } x \leq \frac{1-\gamma}{2} \\ f_{\mathcal{C}}(x)=\beta & \text { if } \frac{1-\gamma}{2}<x<\frac{1+\gamma}{2} \\ f_{\mathcal{R}}(x)=2 \alpha(1-x) & \text { if } x \geq \frac{1+\gamma}{2}\end{cases}
$$

as shown in Fig. 1 and is denoted in the following as the discontinuous flat top tent map. The parameters $\alpha, \beta$ and $\gamma$ refer to the tent tip height, the height and the width of the constant interval, respectively. As one can see, the discontinuous flat top tent map represents an extension of the usual tent map

$$
x_{n+1}=f\left(x_{n}\right), \quad f(x)= \begin{cases}f_{\mathcal{L}}(x)=2 \alpha x & \text { if } x \leq \frac{1}{2} \\ f_{\mathcal{R}}(x)=2 \alpha(1-x) & \text { if } x>\frac{1}{2}\end{cases}
$$

with an additional partition $] \frac{1-\gamma}{2}, \frac{1+\gamma}{2}[$ where the function $f$ has a constant value. Note that a similar extension was already considered in [20]. However, in this work the value of the function on this partition was set to a value outside the interval $[0,1]$, so that every orbit reaching this partition becomes divergent. By contrast to this, we consider the values on this partition (given by the parameter $\beta$ ) which do not lead to divergence.

In the following, the dynamical properties of this map are investigated in the 2D parameter plane $(\alpha, \beta)$ for $\alpha \in(1 / 2, \infty), \beta \in[0,1]$ and arbitrary but fixed $\gamma \in(0,1)$. All analytical and topological arguments are 


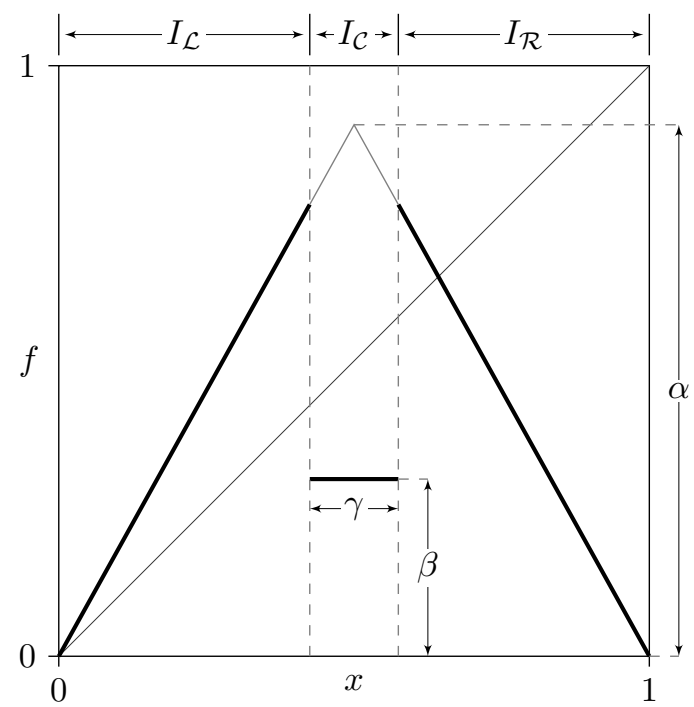

Figure 1. System function of the discontinuous flat top tent map given by Eq. (1).

independent of the particular choice of $\gamma$, i.e., the system is structurally stable with respect to this parameter in the given range. For numerical results we choose $\gamma=0.1$ for consistency.

Fig. 2 shows four bifurcation and period diagrams for fixed $\alpha$ and varying $\beta$ :

a) For $\alpha=0.6$, we can see an interval where two symmetric period-1 incrementing cascades exist, which accumulate at the boundaries of $\beta \in[0,1]$.

b) For $\alpha=0.75$, the scenario as in a) is interrupted between any two adjacent periods, by a pair of period-2 incrementing cascades accumulating at the same point in the middle.

c) For $\alpha=0.85$, the scenario as in b) is interrupted between any two adjacent periods, by a pair of period- 4 incrementing cascades.

d) For $\alpha=0.9$, the scenario is already much further developed and the diagram already demonstrates the overall complexity of the bifurcation structure.

Due to this series of cumulative period incrementing cascades, the complete scenario is referred to as "nested period incrementing".

The bifurcation structure in the plane $(\alpha, \beta)$, as shown in Fig. 3, displays for increasing values of $\alpha$ the successive appearance of additional periodic regions in the form of border collision induced codimension-2 bifurcations denoted as "explosions" or "big bang bifurcations". These occur at distinct values of $\alpha$ between each two adjacent regions. This process accumulates at the value $\alpha^{\star}=1 /(1-\gamma)$, above which all possible orbits, that exist in the discontinuous flat top tent map, are observable in a particular order for varying $\beta \in[0,1]$. We refer to this as the full 1D bifurcation scenario, which is described in Sec. 1.2. For lower values of $\alpha$, only subsets of the full scenario can be observed, as we will show in Sec. 1.4.

We study the system's dynamical properties using a symbolic notation based on the letters $\mathcal{L}, \mathcal{C}$ and $\mathcal{R}$, corresponding to the three disjoint partitions

$$
I_{\mathcal{L}}=\left[0, \frac{1-\gamma}{2}\right], \quad I_{\mathcal{C}}=\left(\frac{1-\gamma}{2}, \frac{1+\gamma}{2}\right), \quad I_{\mathcal{R}}=\left[\frac{1+\gamma}{2}, 1\right]
$$


of the unit interval, as shown in Fig. 1 . More precisely, to each $n$-periodic orbit $\mathcal{O}=\left(x_{0}, x_{1}, \ldots, x_{n-1}\right)$ we define the corresponding symbolic sequence $\sigma=\sigma_{0} \sigma_{1} \ldots \sigma_{n-1}$, such that

$$
\sigma_{i}= \begin{cases}\mathcal{L} & \text { if } x_{i} \in I_{\mathcal{L}} \\ \mathcal{C} & \text { if } x_{i} \in I_{\mathcal{C}} \\ \mathcal{R} & \text { if } x_{i} \in I_{\mathcal{R}}\end{cases}
$$

and denote the corresponding periodic orbit as $\mathcal{O}_{\sigma}$. In the following, the bifurcation scenarios consisting of the stable periodic orbits will be explained and investigated by means of these symbolic sequences.

\subsection{Behavior for $\alpha=\alpha^{\star}$}

To get a first intuitive understanding of the behavior of the discontinuous flat top tent map, let us state how the function branches $f_{\mathcal{L}}, f_{\mathcal{C}}$ and $f_{\mathcal{R}}$ determine the form of periodic orbits. In the considered parameter plane, the dynamics is governed by the following three mechanisms:

M1: a channel in the left partition, originating from the unstable fixed point $\mathcal{O}_{\mathcal{L}}=0$. Each orbit entering the left partition remains in the channel formed by the system function and the principal bisector for a number of iteration steps until it leaves the channel.

M2: a swirl in the right partition, originating from the unstable fixed point $\mathcal{O}_{\mathcal{R}}=\frac{2 \alpha}{2 \alpha+1}$ (if the fixed point exists). Each orbit entering the right partition "rotates" around this point for a number of iteration steps until it leaves the swirl.

M3: a 'hard reset' from the center partition. Each orbit entering this partition in step $n$ is immediately reset to the value $x_{n+1}=\beta$.

It is worth noticing that M3 implies that each periodic orbit visiting the center partition is super-stable, which offers certain numerical advantages. In particular, from any initial value located in this partition, for example $x_{0}=\frac{1}{2}$, the system is guaranteed to reach the asymptotic dynamics exactly, within not more than one transient iteration step. It also follows that any periodic orbit can possess no more than one point in the center partition $I_{\mathcal{C}}$ and, correspondingly, the symbolic sequence cannot contain more than one occurrence of the symbol $\mathcal{C}$.

Furthermore, as the slopes of the function branches $f_{\mathcal{L}}$ and $f_{\mathcal{R}}$ are both of magnitude greater than 1 in the considered parameter domain, any periodic orbit without a point in $I_{\mathcal{C}}$ must be unstable. Accordingly, the following statements are equivalent for $\alpha>\frac{1}{2}$ :

$$
\begin{gathered}
\text { "The symbolic sequence } \sigma \text { contains } \\
\text { the symbol } \mathcal{C} \text { exactly once." }
\end{gathered} \Leftrightarrow \quad \begin{gathered}
\text { "The periodic orbit } \mathcal{O}_{\sigma} \text { corresponding } \\
\text { to the sequence } \sigma \text { is stable." }
\end{gathered}
$$

This means that we can uniquely describe each stable $n$-periodic orbit using a symbolic sequence $\sigma$ with the leading symbol $\sigma_{0}=\mathcal{C}$ and $\sigma_{i} \in\{\mathcal{L}, \mathcal{R}\}, i=1 \ldots n-1$. With this definition, $\mathcal{C}$ becomes obsolete as a symbol for the description of stable periodic orbits. Hence, as long as we are dealing with stable periodic orbit, the leading $\mathcal{C}$ can be dropped, since the remaining word consisting solely of symbols $\mathcal{L}$ and $\mathcal{R}$ is, in this case, the only information-carrying part of the symbolic sequence.

However, since in the present work we are dealing both with stable and with unstable periodic orbits we will keep the leading letter $\mathcal{C}$ and shall write all symbolic sequences corresponding to stable periodic orbits in the form $\mathcal{C} \varrho=\mathcal{C} \varrho_{1} \varrho_{2} \ldots \varrho_{n-1}, \varrho_{i} \in\{\mathcal{L}, \mathcal{R}\}$. Note that the subsequence $\varrho$ (without the leading symbol $\mathcal{C}$ ) is used by Metropolis, Stein and Stein in the famous work [12] to describe super-stable periodic orbits, going by the name "pattern". Clearly, by contrast to the symbolic sequences usually used for description of periodic orbits, such "patterns" are not shift-invariant.

Now let us interpret the mechanisms M1-3 introduced earlier with respect to the symbolic sequences of the orbits that they generate. The channel (M1) permits an arbitrary number of steps in the left partition, which corresponds to a subsequence $\mathcal{L} \mathcal{L} \ldots \mathcal{L}$. As the fixed point at the origin is unstable, the orbit is bound to leave the channel at some point. Note that $f_{\mathcal{L}}$ is surjective for $\alpha=\alpha^{\star}$, so the orbit may thereafter land on any 
a)

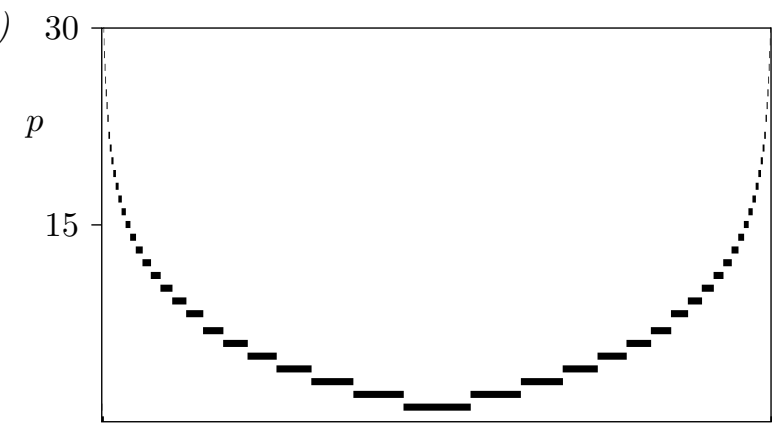

b)

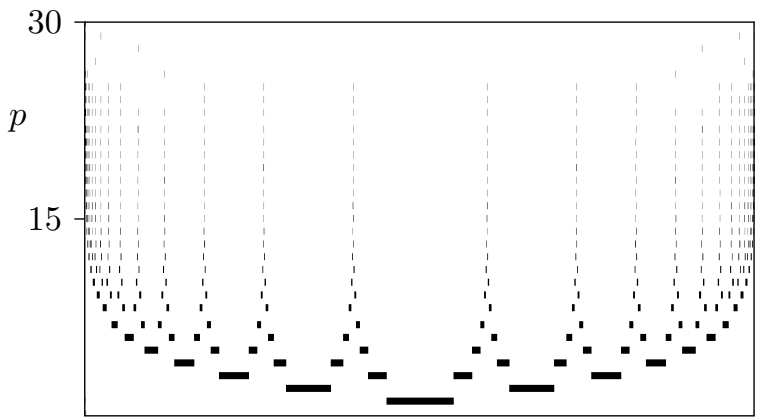

c)

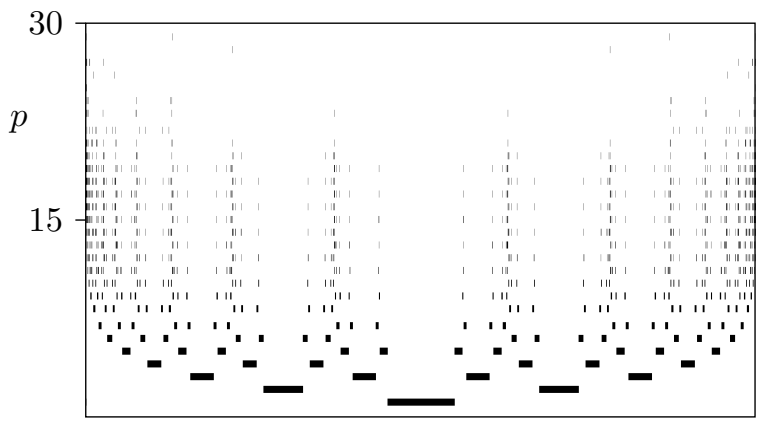

d)

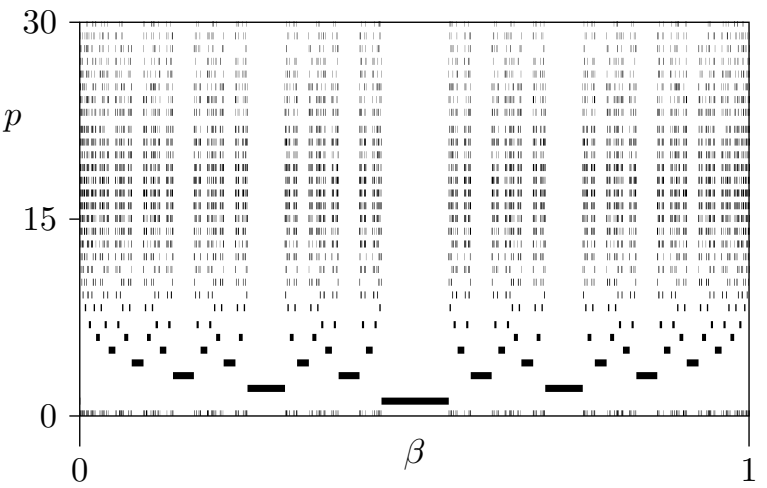

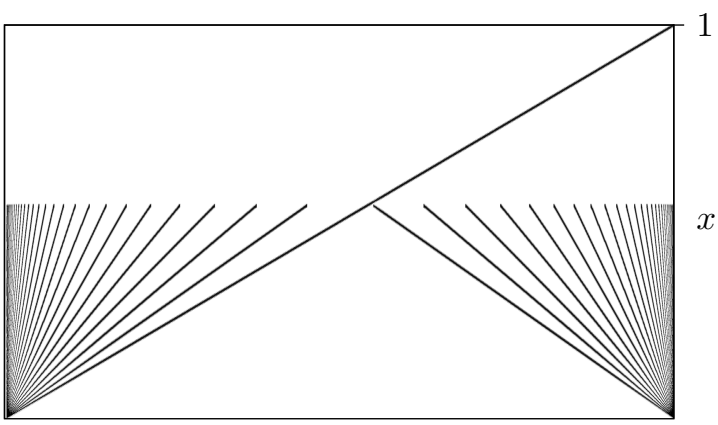
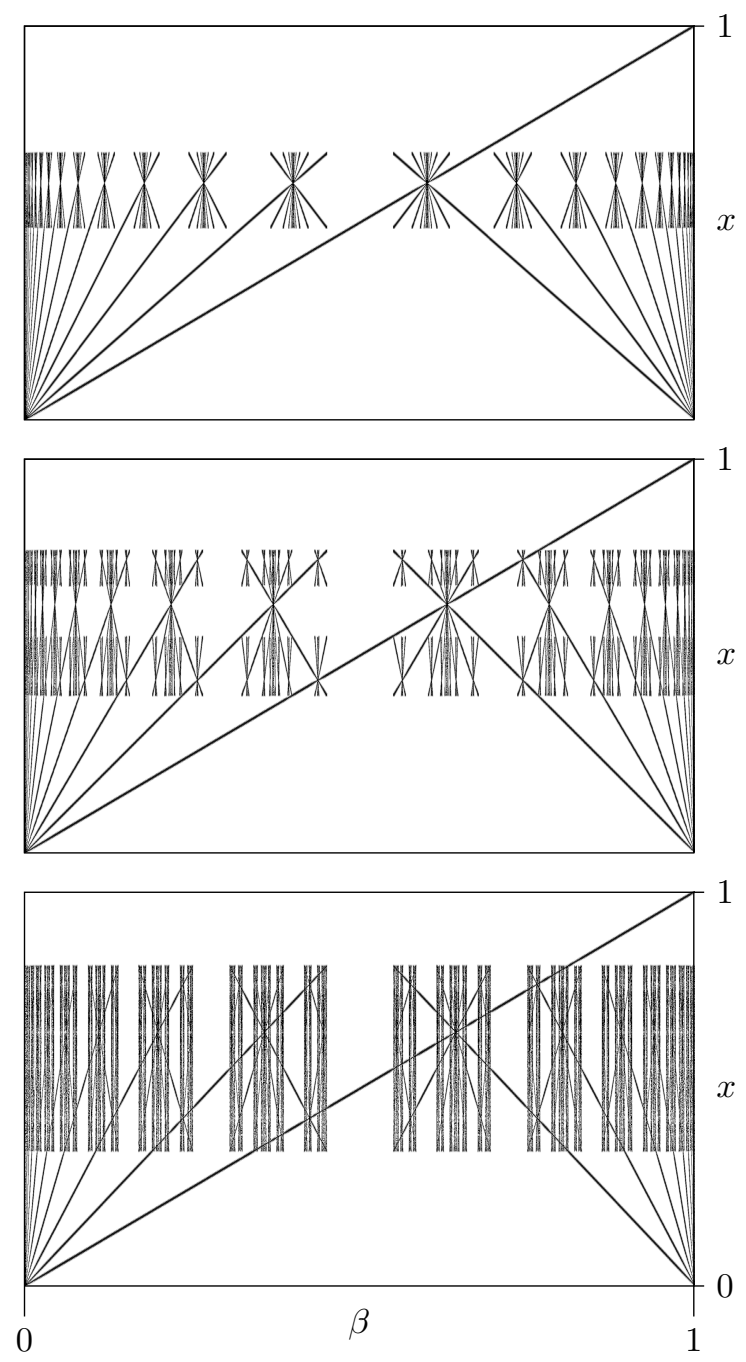

FiguRE 2. Representative examples for period diagrams (left) and the corresponding bifurcation diagrams (right) for $\gamma=0.1$ and different values of $\alpha$ : a) pure period incrementing for $\alpha=0.6, \mathrm{~b})$ pairs of opposed period incrementing cascades with an increment value of two in between for $\alpha=0.75, \mathrm{c}$ ) even further nesting for $\alpha=0.85$. In d) for $\alpha=0.9$, a far more complex scenario has evolved. (The location of these scenarios in the parameter plane $(\alpha, \beta)$ is marked in Fig. 3). 


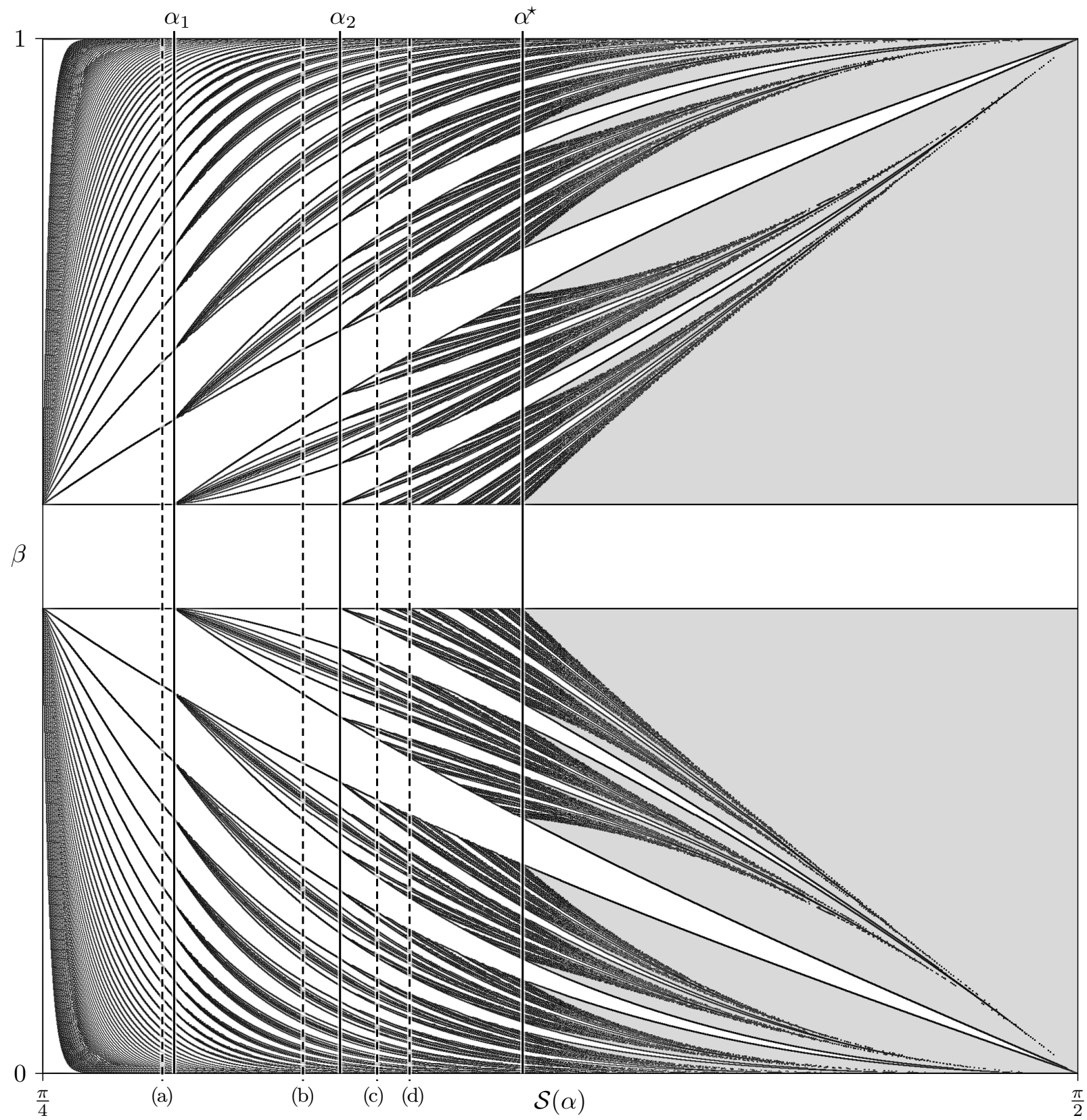

Figure 3. 2D bifurcation structure of the discontinuous flat top tent map (1) in the $(\alpha, \beta)$ plane, for $\gamma=0.1$ and $\alpha \in] \frac{1}{2}, \infty$ [, via the transform $\mathcal{S}(\alpha)=\arctan (2 \alpha)$. At $\alpha_{1}$ the unstable fixed point $\mathcal{O}_{\mathcal{R}}$ emerges (see below) and, at $\alpha_{2}$, the unstable period-2 orbit $\mathcal{O}_{\mathcal{L R}}$. At $\alpha^{\star}$ the escape region emerges. In the gray parameter regions, the typical orbits diverge. The labels (a,b,c,d) indicate the locations of the bifurcation scenarios shown in Fig. 2. 


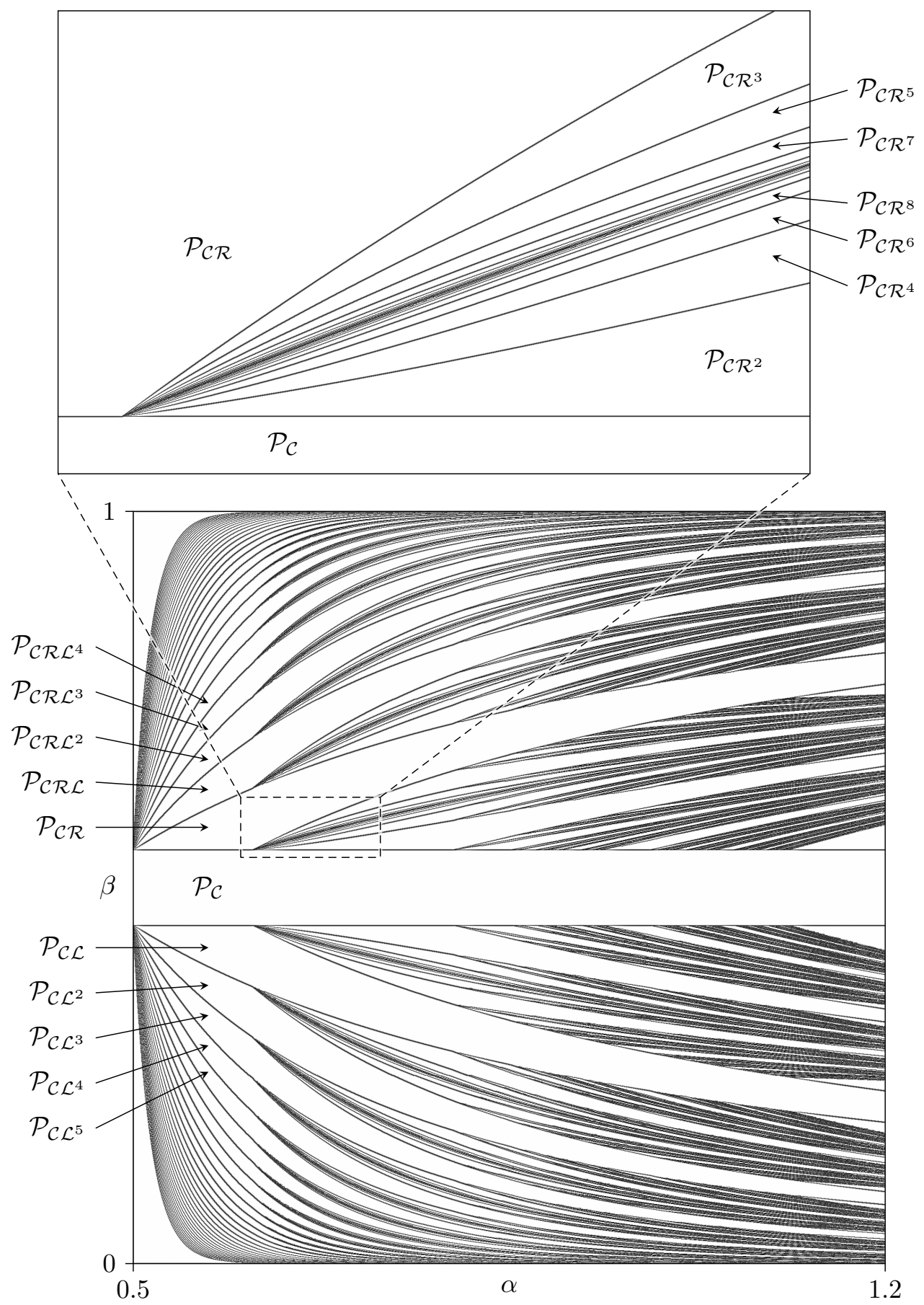

FIgURE 4. The same 2D bifurcation diagram as in Fig. 3 without scaling of the parameter $\alpha$. For a selection of periodic orbits $\mathcal{O}_{\sigma}$, the periodicity regions $\mathcal{P}_{\sigma}$ are explicitly labeled. 
point of either the $\mathcal{C}$-partition - in which case the orbit closes - or of the $\mathcal{R}$-partition. Here the swirl (M2) generates a subsequence $\mathcal{R} \mathcal{R} \ldots \mathcal{R}$, followed by either M1 or M3. The reset mechanism (M3) closes the orbit and determines the value $x_{1}=\beta$, and by that also the first symbol of $\varrho$. Recall that we chose $\sigma_{0}=\mathcal{C}$, i.e., $x_{0} \in I_{\mathcal{C}}$.

It turns out that, in the discontinuous flat top tent map, the interplay of swirl and channel can generate periodic orbits with arbitrary sequences of the letters $\mathcal{L}$ and $\mathcal{R}$. To be precise, for $\alpha=\alpha^{\star}$ and varying $\beta \in[0,1]$, the system produces all $2^{n-1}$ distinct sequences $\varrho \in\{\mathcal{L}, \mathcal{R}\}^{n-1}$ corresponding to different stable $n$-periodic orbits $\mathcal{O}_{\sigma}$ with $\sigma=\mathcal{C} \varrho$.

a)

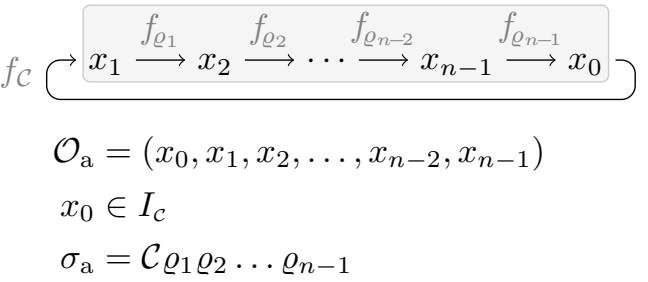

b)

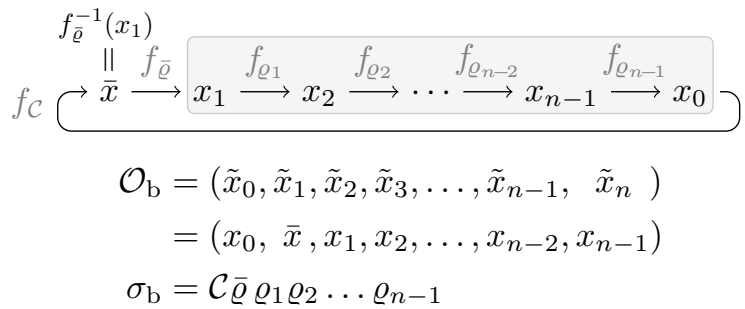

Figure 5. Two periodic orbits of the discontinuous flat top tent map with a preimage relationship in parameter space: the left orbit $\mathcal{O}_{\mathrm{a}}$ exist for some value $\beta_{\mathrm{a}}$, the right orbit $\mathcal{O}_{\mathrm{b}}$ is observed when $\beta$ is set to a preimage $\beta_{\mathrm{b}}=f_{\bar{\varrho}}^{-1}\left(\beta_{\mathrm{a}}\right)$ of the original parameter value, with $\bar{\varrho} \in\{\mathcal{L}, \mathcal{R}\}$.

This fact is readily explained. Fig. 5a shows an arbitrary stable $n$-periodic orbit $\mathcal{O}_{\mathrm{a}}=\left(x_{0}, \ldots, x_{n-1}\right)$ of the discontinuous flat top tent map, assumed to exist for $\alpha=\alpha^{\star}$ and appropriate $\beta=\beta_{\mathrm{a}}$. Let us also assume that, in keeping with the convention introduced above, the first point $x_{0}$ lies in the flat interval $I_{\mathcal{C}}$. Of course, this implies that the location of the orbit's second point depends solely on the parameter $\beta$, that is $x_{1}=f_{\mathcal{C}}\left(x_{0}\right)=\beta_{\mathrm{a}}$. Now, we exploit the fact that this value can be varied independently of other parameters.

With a suitable choice of $\beta$, namely $\beta_{\mathrm{b}}=f_{\bar{\varrho}}^{-1}\left(\beta_{\mathrm{a}}\right)$ with either $\bar{\varrho}=\mathcal{L}$ or $\bar{\varrho}=\mathcal{R}$, a new stable periodic orbit $\mathcal{O}_{\mathrm{b}}=\left(\tilde{x}_{0}, \tilde{x}_{1}, \ldots\right)$ can be found, as shown in Fig. 5b. The basic assumption $\tilde{x}_{0}=x_{0} \in I_{\mathcal{C}}$ yields $\tilde{x}_{1}=\beta_{\mathrm{b}} \in I_{\bar{\varrho}}$ and consequently $\tilde{x}_{2}=f_{\bar{\varrho}}\left(\beta_{\mathrm{b}}\right)=\beta_{\mathrm{a}}$. This point, however, coincides with the second point $x_{1}$ of $\mathcal{O}_{\mathrm{a}}$. Here the orbit evolves according to one of the branches $f_{\mathcal{L}} / f_{\mathcal{R}}$, which are of course independent of $\beta$, so the remainders of both orbits behave identically, i.e.,

$$
\tilde{x}_{k}=x_{k-1} \text { for } k=2, \ldots, n \text { and } f\left(\tilde{x}_{n}\right)=f\left(x_{n-1}\right)=x_{0}=\tilde{x}_{0}
$$

The result is that the $(n+1)$-periodic orbit $\mathcal{O}_{\mathrm{b}}$ can be obtained from $\mathcal{O}_{\mathrm{a}}$ simply by inserting one point $\bar{x}=$ $f_{\bar{Q}}^{-1}\left(x_{1}\right)$ after the point $x_{0} \in I_{\mathcal{C}}$. Accordingly, the symbolic sequences of both orbits are also identical up to one additional symbol $\bar{\varrho}$, as seen in Fig. 5 .

This means that, for every ${ }^{2}$ existing stable orbit with period $n$ and the corresponding symbolic sequence $\sigma=\mathcal{C} \varrho$, two orbits with period $n+1$ can be found, corresponding to the sequences $\sigma^{\prime}=\mathcal{C} \mathcal{L} \varrho$ and $\sigma^{\prime \prime}=\mathcal{C} \mathcal{R} \varrho$. Applying this procedure recursively, beginning with the case of the stable fixed point $\mathcal{O}_{\mathcal{C}}$ located in the middle partition, proves the existence of all possible orbits as stated above. For the symbolic sequence of the fixed point we write $\sigma=\mathcal{C} \varrho=\mathcal{C}$ and denote $\varrho=\varepsilon$ as the empty word for reasons of consistency.

As a consequence, it is now easy to identify the existence region of any stable periodic orbit at $\alpha=\alpha^{\star}$, given its symbolic sequence. We know that the fixed point $\mathcal{O}_{\mathcal{C}}$ exists for $\beta \in I_{\mathcal{C}}$, so by reasoning as above the existence condition for an arbitrary orbit $\mathcal{O}_{\mathcal{C} \varrho}$ results in

$$
\beta \in U_{\mathcal{C} \varrho} \quad \text { with } \quad U_{\mathcal{C} \varrho}=f_{\varrho}^{-1}=f_{\varrho_{1}}^{-1} \circ f_{\varrho_{2}}^{-1} \circ \cdots \circ f_{\varrho_{n-1}}^{-1}\left(I_{\mathcal{C}}\right) .
$$

\footnotetext{
${ }^{2}$ At least this is true for $\alpha \geq \alpha^{\star}$, where every point $x \in[0,1]$ has two preimages $f_{\mathcal{L}}^{-1}(x)$ and $f_{\mathcal{R}}^{-1}(x)$. With smaller values of $\alpha$, these preimages do not necessarily exist for all these points.
} 
We can also make a more compact statement about the locations of these stable periodic orbits in the parameter space. For this reason we number all sequences of the same period according to the order of the corresponding orbits along the $\beta$-axis. Let us denote the sequence corresponding to the $i$-th orbit of period $n$ as $\sigma_{i}^{n}$. So for $n=1$ there is one sequence $\sigma_{1}^{1}=\mathcal{C}$, for $n=2$ there are two sequences $\sigma_{1}^{2}=\mathcal{C} \mathcal{L}$ and $\sigma_{2}^{2}=\mathcal{C} \mathcal{R}$, and so forth. In general, of all $2^{n-1}$ sequences corresponding to the orbits of period $n$, the sequence whose corresponding orbit appears at the smallest value of $\beta$ is referred to as $\sigma_{1}^{n}$, and the one appearing at the largest value is $\sigma_{2^{n-1}}^{n}$. Note also that the location of the preimages satisfies

$$
\left|\frac{1}{2}-f_{\mathcal{L}}^{-1}(x)\right|=\left|\frac{1}{2}-f_{\mathcal{R}}^{-1}(x)\right|=\frac{1}{2}\left(1-\frac{x}{\alpha^{\star}}\right),
$$

i.e. the distance of the preimages to the point $\frac{1}{2}$ decreases linearly in $x$. This means that the smallest $x$ has the "outermost", and the largest $x$ the "innermost" preimages. As already shown, the parameter change $\beta \mapsto f_{\varrho}^{-1}(\beta), \bar{\varrho} \in\{\mathcal{L}, \mathcal{R}\}$ is equivalent to the sequence change $\mathcal{C} \varrho \mapsto \mathcal{C} \varrho \varrho$. The location on the $\beta$-axis of the two period- $(n+1)$ orbits corresponding to these two sequences $\mathcal{C} \mathcal{L} \varrho$ and $\mathcal{C} \mathcal{R} \varrho$ can be explained easily based on Eq. (7). For example, as the sequence $\sigma_{1}^{n}=\mathcal{C} \varrho_{1}^{n}$ has the smallest $\beta$-value among all sequences corresponding to period- $n$ orbits, so the sequence $\mathcal{C} \mathcal{L} \varrho_{1}^{n}$ has the smallest $\beta$-value among all $2^{n}$ sequences corresponding to period- $(n+1)$ orbits, that means $\mathcal{C} \mathcal{L} \varrho_{1}^{n}=\sigma_{1}^{n+1}$. Similarly, the sequence $\mathcal{C} \mathcal{R} \varrho_{1}^{n}$ has the largest $\beta$-value among all sequences corresponding to period- $(n+1)$ orbits, that means $\mathcal{C} \mathcal{R} \varrho_{1}^{n}=\sigma_{2^{n}}^{n+1}$. In general, all $2^{n}$ sequences corresponding to period- $(n+1)$ orbits can be generated from the $2^{n-1}$ sequences corresponding to period- $n$ orbits recursively according to the following rules.

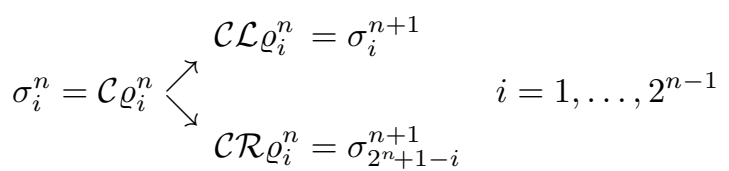

Again, the indexes $i=1, \ldots, 2^{n}$ of the sequences $\sigma_{i}^{n+1}$ define the order of the corresponding orbits along the $\beta$-axis. In the following this order will be denoted as the $\beta$-ordering of the sequences.

Note that the rules given by Eq. (8) generate the $\beta$-ordering of the sequences corresponding to orbits with a certain period, but without making a direct statement about their relative ordering to the sequences of other periods. However, what we need in order to explain the bifurcation structure occurring at $\alpha=\alpha^{\star}$ is the $\beta$-ordering of all sequences, regardless of their period.

However, we can easily retrace the partitioning of the $\beta$-axis into periodicity regions with certain symbolic sequences. The locations of these regions are given by Eq. (6), as already stated. By applying this equation one symbol at a time, all regions can be calculated in a recursive manner: in the first step, it is only known that the interval $[0,1]$ is subdivided (in $\beta$-ordering) into the three partitions $I_{\mathcal{L}}, I_{\mathcal{C}}$ and $I_{\mathcal{R}}$. We now denote these partitions as $P_{\mathcal{C} \mathcal{L}}, U_{\mathcal{C}}$ and $P_{\mathcal{C} \mathcal{R}}$, respectively. $U_{\mathcal{C}}=I_{\mathcal{C}}$ is of course the existence region of the stable fixed point with the sequence $\sigma=\mathcal{C}$, whereas the partitions $P_{\mathcal{C} \mathcal{L}}=I_{\mathcal{L}}$ and $P_{\mathcal{C} \mathcal{R}}=I_{\mathcal{R}}$ are the regions where all periodic orbits have a common prefix $\mathcal{C} \mathcal{L} / \mathcal{C} \mathcal{R}$, respectively. For instance, any stable periodic orbit existing for $\beta \in P_{\mathcal{C} \mathcal{L}}$ has the point $x_{1} \in I_{\mathcal{L}}$ and therefore must have a symbolic sequence beginning with $\mathcal{C} \mathcal{L}$, possibly followed by further symbols.

Now, for $\alpha=\alpha^{\star}$ the function $f_{\mathcal{L}}^{-1}$ maps the interval $[0,1]$ linearly onto the partition $P_{\mathcal{C} \mathcal{L}}$, which is therefore further subdivided into the following three partitions (one periodicity region and two common-prefix regions):

$$
P_{\mathcal{C} \mathcal{L}^{2}}=f_{\mathcal{L}}^{-1}\left(I_{\mathcal{L}}\right), \quad U_{\mathcal{C} \mathcal{L}}=f_{\mathcal{L}}^{-1}\left(I_{\mathcal{C}}\right), \quad P_{\mathcal{C} \mathcal{R}}=f_{\mathcal{L}}^{-1}\left(I_{\mathcal{R}}\right)
$$

For the right partition $P_{\mathcal{C} \mathcal{R}}$, the situation is the same, but as $f_{\mathcal{R}}$ has a negative slope, the subdivision occurs in the opposite order:

$$
P_{\mathcal{C} \mathcal{R}^{2}}=f_{\mathcal{R}}^{-1}\left(I_{\mathcal{R}}\right), \quad U_{\mathcal{C R}}=f_{\mathcal{R}}^{-1}\left(I_{\mathcal{C}}\right), \quad P_{\mathcal{C R} \mathcal{L}}=f_{\mathcal{R}}^{-1}\left(I_{\mathcal{L}}\right)
$$


In summary, this procedure so far yields the following $\beta$-ordering of periodicity and common-prefix regions:

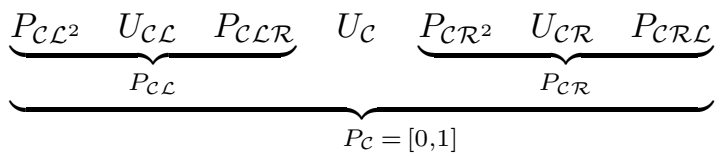

Carried out ad infinitum, this procedure is equivalent to arranging all sequences in a binary tree structure, where each level contains the sequences of the corresponding period. The relationship in this tree is such that the children of a particular node are the sequences of the next higher period, which are located the nearest along $\beta$ in parameter space. The tree of symbolic sequences up to period $n=6$ is shown in Fig. 6 . This tree is generated by recursive application of the following rules:

S1 Start by defining the root node with the sequence $\sigma=\mathcal{C}$.

S2 To each node where $\sigma$ contains an $e v e n^{3}$ number of $\mathcal{R}$ s (we say, $\sigma$ has an even $\mathcal{R}$-parity), add a left child node with the sequence $\sigma \mathcal{L}$, and a right child with the sequence $\sigma \mathcal{R}$.

S3 To each node where $\sigma$ contains an odd number of $\mathcal{R}$ s (we say, $\sigma$ has an odd $\mathcal{R}$-parity), add a left child with the sequence $\sigma \mathcal{R}$, and a right child with the sequence $\sigma \mathcal{L}$.

We denote these rules as the Suffix Rules. Note that these rules add a letter at the end of a sequence, whereas the rules given by Eq. (8) require insertion of a letter right after the symbol $\mathcal{C}$. It is also worth emphasizing that the Suffix Rules differ from Eq. (8), in that the tree reproduces the $\beta$-ordering of sequences corresponding to orbits up to a certain period. It is clear that by recursive appending of both $\mathcal{L}$ and $\mathcal{R}$, all sequences must be produced. Note further that these rules were already mentioned in [1], in the context of the logistic map, where the location of symbolic sequences along the interval of initial values is investigated, leading to a subdivision of the parameter space similar to the common-prefix partitioning given by Eq. (11). Although not surprisingly, this fact points out an intimate relationship between the discontinuous flat top tent map and the logistic map.

It is now an easy task to identify the region of parameter space where stable periodic orbits of the form $\sigma=\mathcal{C} \varrho$ occur. This region is of course given by the union of all preimages of the middle partition $I_{\mathcal{C}}$ :

$$
S=\bigcup_{n=0}^{\infty} f^{-n}\left(I_{\mathcal{C}}\right)
$$

which is everywhere dense in $[0,1]$, i.e., the closure of $S$ is $\bar{S}=[0,1]$. This means that the discontinuous flat top tent map for $\alpha=\alpha^{\star}$ possesses a stable periodic orbit for any parameter value $\beta \in[0,1]$ except on a set of Lebesgue measure zero. Although we constructed the set $S$ in parameter space $(\beta \in S)$, we can also regard this set in state space as the set of all points that eventually get mapped to $I_{\mathcal{C}}$. In order to avoid confusion, we shall in the following refer to the set $S$ in parameter space, $\beta \in S$, whereas the notation $\widetilde{S}$ is used for the same set in state space, $x \in \widetilde{S}$.

We can immediately see that $\widetilde{S}$ belongs to the stable set of any orbit which has a point in $I_{\mathcal{C}}$. This implies that the stable periodic orbit $\mathcal{O}_{\mathcal{C}}$ which exists for a certain parameter value is the unique attractor of the discontinuous flat top tent map attracting almost all initial values $x_{0} \in[0,1]$. Note that this statement holds only for $\frac{1}{2}<\alpha \leq \alpha^{\star}$. For $\alpha>\alpha^{\star}$ the set $\widetilde{S}$ is no longer dense in $[0,1]$ and the orbits for the initial values $x_{0} \in[0,1] \backslash C l(\widetilde{S})$ diverge, whereby $C l(\widetilde{S})$ denotes the closure of $\widetilde{S}$.

Interestingly, the construction of $S$ implies $^{4}$ that its complement $M=[0,1] \backslash S$ is a Cantor set $^{5}$. Equivalently to the sets $S$ and $\widetilde{S}$, we shall distinguish in the following between sets $M$ in parameter space and $\widetilde{M}=[0,1] \backslash \widetilde{S}$ in state space. In parameter space, the set $M$ consists of the accumulation points of the nested period incrementing scenario. As the periods of the stable periodic orbits close to any point in $M$ tend to infinity, the nature of

\footnotetext{
3 including zero

${ }^{4}$ Not only for $\alpha=\alpha^{\star}$ but for any $\alpha>\alpha_{\infty}$ (see $\left.\S 2.1\right)$.

${ }^{5}$ For example, in the special case $\gamma=1 / 3$ it is the well-known middle third Cantor set.
} 


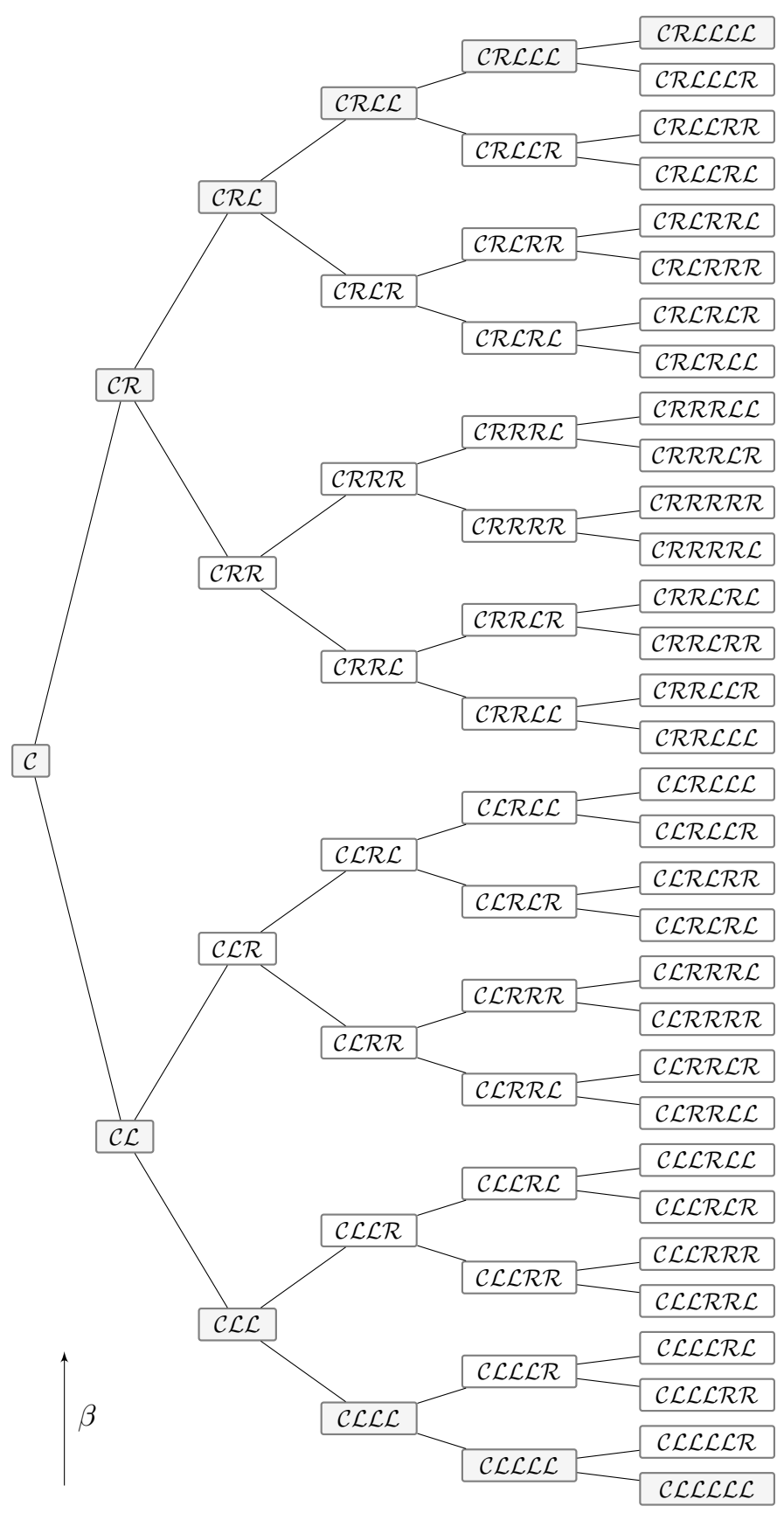

Figure 6 . The full binary tree of symbolic sequences corresponding to stable periodic orbits up to period 6 . The $\beta$-ordering is indicated by the arrow. The orbits corresponding to the outermost sequences exist for $\alpha<\alpha_{1}$ (see Figs. 2a and 4). 
the dynamical behavior in the limit $\beta \in M$ is of some interest. This is briefly investigated in the following subsection. In the state space the set $\widetilde{M}$ contains unstable periodic orbits, their stable sets, as well as aperiodic orbits.

\subsection{Milnor Attractors}

So far we considered only stable periodic orbits. We already know of two particular unstable periodic orbits, namely the fixed points $\mathcal{O}_{\mathcal{L}}=0$ and $\mathcal{O}_{\mathcal{R}}=2 \alpha /(2 \alpha+1)$. It can be shown that, for $\alpha=\alpha^{\star}$, the discontinuous flat top tent map actually possesses a countable infinity of unstable periodic orbits, as well as an uncountable infinity of unstable aperiodic orbits, which can be uniquely identified by corresponding (respectively terminating and non-terminating) symbolic sequences.

From the preceding section we know which orbits exist for any $\beta \in S$. Now we can also state what happens if $\beta$ lies within the Cantor set $M$. For this, we consider the behavior of the tent map (2) for $\alpha=\alpha^{\star}$. Note that $\alpha^{\star}=1 /(1-\gamma)>1$, so whereas (1) and (2) are identical on the partitions $I_{\mathcal{L}}$ and $I_{\mathcal{R}}$, the tent map possesses an escape interval at $I_{\mathcal{C}}$. That means that all orbits started at $x_{0} \in \widetilde{S}$, i.e., on the escape interval or one of its preimages, diverge under the dynamics of the tent map. For all other orbits with $x_{0} \in \widetilde{M}$, the behavior of the tent map and the discontinuous flat top tent map is identical. It is known that the non-diverging orbits of the tent map for $\alpha>1$ can have all possible symbolic sequences consisting of $\mathcal{L}$ and $\mathcal{R}$.

It is worth noting that the behavior in the generic case $\beta \in S$ is quite different from the behavior in the special case $\beta \in M$. For $\beta \in S$, an orbit started at a typical initial value $x_{0} \in \widetilde{S}$ converges to the unique stable periodic orbit $\mathcal{O}_{\sigma}$ which necessarily contains a point $x_{0}^{\sigma} \in I_{\mathcal{C}}$ and - with the exception of the fixed point $\mathcal{O}_{\mathcal{C}}-$ also the point $x_{1}^{\sigma}=\beta$ (in other words, the point $x=\beta$ is periodic in this case). All unstable periodic orbits located in the set $\widetilde{M}$ coexist with this unique stable periodic orbit. Also, the stable set $W^{s}$ of each unstable orbit is a subset of $\widetilde{M}$. So in this case the stable set of each unstable orbit is of zero measure.

For $\beta \in M$, an orbit started at a typical initial value $x_{0} \in \widetilde{S}$ will be mapped to some point $x \in \widetilde{M}$ and eventually to one of the orbits contained in this set, which is either an unstable periodic or an aperiodic orbit. Although these two cases are similar, it is worth to distinguish between them.

If the point $x=\beta \in \widetilde{M}$ is pre-periodic and will be mapped (directly or after some iteration steps) to a point of an unstable periodic orbit, this unstable orbit attracts a set of points of positive Lebesgue measure. Indeed, the set of initial values converging to this particular unstable orbit includes $\widetilde{S}$ and is therefore dense in $[0,1]$. Invariant sets of this kind, commonly known as Milnor attractors (sometimes denoted also as weak attractors), are examined e.g. in [13].

Generally, these invariant sets have both the stable and the unstable set of positive Lebesgue measure. By contrast to regular attractors (given by stable periodic orbits), the Milnor attractors are not robust, since the domains of these attractors in the $(\alpha, \beta)$ parameter plane are given by sets of curves and not by regions (see $\S \S 2.2$ and 2.5 for details).

In the case that $x=\beta \in \widetilde{M}$ is mapped to a point of an aperiodic orbit (that means, the point $x=\beta$ is neither periodic nor pre-periodic), the situation is similar. The main difference in this case is that this aperiodic orbit does not represent a closed set and therefore can be denoted as some kind of attracting set (because every typical initial condition $x_{0} \in \widetilde{S}$ converges to this orbit) but not as an attractor. However, its closure (the $\omega$-limit set of its points) represents a Milnor attractor. Another difference regards the bifurcation structures in the $(\alpha, \beta)$ parameter plane: As we will see below, in the parameter space the Milnor attractors are located at the accumulation points of the period incrementing cascades, whereas the attracting set mentioned above are not.

\subsection{Behavior for $\alpha<\alpha^{\star}$}

Above we summarized the behavior of the discontinuous flat top tent map at $\alpha=\alpha^{\star}$. In the case $\alpha>\alpha^{\star}$ the situation is similar: each attractor existing at $\alpha=\alpha^{\star}$ exists also for any $\alpha>\alpha^{\star}$, and the only additional phenomenon possible in this case is divergent dynamics. Regarding the situation for $\alpha<\alpha^{\star}$ it is immediately 
clear from Figs. 2 and 3 that the number of periodic orbits which can be observed by varying $\beta$ decreases with decreasing $\alpha$. This can be explained as follows. The stable periodic orbit $\mathcal{O}_{\sigma}$ corresponding to the symbolic sequence $\sigma=\sigma_{0} \sigma_{1} \ldots \sigma_{n-1}$ with $\sigma_{0}=\mathcal{C}$ (or more precisely, its first point $x_{0}^{\sigma}$ ) can be found using the fixed point equation

$$
f_{\sigma}\left(x_{0}^{\sigma}\right):=f_{\varrho_{n-1}} \circ f_{\varrho_{n-2}} \circ \cdots \circ f_{\varrho_{2}} \circ f_{\varrho_{1}}(\underbrace{f_{\mathcal{C}}\left(x_{0}^{\sigma}\right)}_{\beta})=x_{0}^{\sigma}
$$

Here we use again (as in $\$ 1.2$ ) the notation $\sigma=\mathcal{C} \varrho$ with $\varrho_{i}=\sigma_{i}, i=1, \ldots, n-1$. Clearly, for a given combination of the parameters $\alpha$ and $\beta$ the orbit $\mathcal{O}_{\sigma}$ exists only if all points calculated in this way are located in the corresponding partitions, that means $x_{0}^{\sigma}$ in $I_{\mathcal{C}}, x_{1}^{\sigma}=f_{\mathcal{C}}\left(x_{0}^{\sigma}\right)=\beta$ in the partition corresponding to the letter $\sigma_{1}$, and so on. Therefore, to verify whether all these conditions are fulfilled for a given combination of $\alpha$ and $\beta$ we can calculate the rank- $(n-1)$ preimage of the middle partition, given by the interval $U_{\mathcal{C} \varrho}$ defined by Eq. (6). As stated in $\S 1.2$, the orbit $\mathcal{O}_{\sigma}$ exists only if the point $x_{1}^{\sigma}=\beta$ belongs to the interval $U_{\mathcal{C} \varrho}$. Moreover, note that for all $i=1, \ldots, n-1$ the letters $\varrho_{i}$ are in $\{\mathcal{L}, \mathcal{R}\}$, and hence $f_{\varrho}^{-1}\left(I_{\mathcal{C}}\right)$ depends on the parameter $\alpha$ but not $\beta$. Therefore, assuming that the interval $f_{\varrho}^{-1}\left(I_{\mathcal{C}}\right)$ is not empty, one can set the parameter $\beta$ to a value in this interval, and this guarantees that the orbit $\mathcal{O}_{\sigma}$ exists. In other words, the condition

$$
\beta \in f_{\varrho}^{-1}\left(I_{\mathcal{C}}\right)
$$

represents the existence condition of the stable periodic orbit $\mathcal{O}_{\sigma}$ and the boundaries of this interval correspond to the border collision bifurcations causing this orbit to disappear.

As one can see, the question whether the stable periodic orbit $\mathcal{O}_{\sigma}$ exists at a given value of $\alpha$ reduces to the question whether the interval $f_{\varrho}^{-1}\left(I_{\mathcal{C}}\right)$ is non-empty. As at $\alpha=\alpha^{\star}$ the functions $f_{\mathcal{L}}$ and $f_{\mathcal{R}}$ map their corresponding partitions onto the complete interval $[0,1]$ :

$$
f_{\mathcal{L}}: I_{\mathcal{L}} \mapsto[0,1], \quad f_{\mathcal{R}}: I_{\mathcal{R}} \mapsto[0,1]
$$

each point $x \in[0,1]$ has at $\alpha=\alpha^{\star}$ both the left and the right preimage, given by

$$
f_{\mathcal{L}}^{-1}(x)=\frac{x}{2 \alpha} \quad \text { and } \quad f_{\mathcal{R}}^{-1}(x)=1-\frac{x}{2 \alpha},
$$

respectively. Due to this, at $\alpha=\alpha^{\star}$ the interval $f_{\varrho}^{-1}\left(I_{\mathcal{C}}\right)$ is non-empty for any sequence $\varrho$, and hence the stable periodic orbit $\mathcal{O}_{\sigma}$ exists in a non-empty range of the parameter $\beta$. By contrast, for $\alpha<\alpha^{\star}$ the functions $f_{\mathcal{L}}$ and $f_{\mathcal{R}}$ map their corresponding partitions not on the complete interval $[0,1]$ but only on $[0, \alpha(1-\gamma)]$ :

$$
f_{\mathcal{L}}: I_{\mathcal{L}} \mapsto[0, \alpha(1-\gamma)], \quad f_{\mathcal{R}}: I_{\mathcal{R}} \mapsto[0, \alpha(1-\gamma)]
$$

Therefore, in this case Eq. (16) is valid only for $x \in[0, \alpha(1-\gamma)]$, whereas the points in the remaining interval $(\alpha(1-\gamma), 1]$ do not have any preimages by $f_{\mathcal{L}}$ and $f_{\mathcal{R}}$ :

$$
f_{\mathcal{L}}^{-1}(x)=\left\{\begin{array}{ll}
\frac{x}{2 \alpha} & \text { if } x \in[0, \alpha(1-\gamma)] \\
\text { undefined } & \text { otherwise }
\end{array} \text { and } f_{\mathcal{R}}^{-1}(x)= \begin{cases}1-\frac{x}{2 \alpha} & \text { if } x \in[0, \alpha(1-\gamma)] \\
\text { undefined } & \text { otherwise }\end{cases}\right.
$$

This leads to the fact that for a given $\alpha<\alpha^{\star}$ and a given $\sigma=\mathcal{C} \varrho$, the interval $f_{\varrho}^{-1}\left(I_{\mathcal{C}}\right)$ may be empty, so that the stable periodic orbit $\mathcal{O}_{\sigma}$ does not exist at this value of $\alpha$.

Let us illustrate the described situation for some value $\frac{1}{2}<\alpha<\alpha_{1}$ with

$$
\alpha_{1}=\frac{1}{2} \frac{1+\gamma}{1-\gamma}
$$


As one can clearly see in Fig. 3 the discontinuous flat top tent map has in this case the most structure of the parameter space, organized by two period incrementing cascades (see Fig. $2 a$ ).

Clearly (as for any $\alpha$ ) for $\beta$ in the middle partition $I_{\mathcal{C}}=\left(\frac{1-\gamma}{2}, \frac{1+\gamma}{2}\right)$ we have the existence region of the fixed point $\mathcal{O}_{\mathcal{C}}$. For the middle partition we can calculate the left and the right preimages, which are given by

$$
f_{\mathcal{L}}^{-1}\left(I_{\mathcal{C}}\right)=\left(\frac{1-\gamma}{4 \alpha}, \frac{1-\gamma}{2}\right] \quad \text { and } \quad f_{\mathcal{R}}^{-1}\left(I_{\mathcal{C}}\right)=\left[\frac{1+\gamma}{2}, 1-\frac{1-\gamma}{4 \alpha}\right)
$$

respectively. Note that the condition $1 / 2<\alpha<\alpha_{1}$ implies that the preimages exist not for all points on the middle partition but only for $x \in[(1-\gamma) / 2, \alpha(1+\gamma)] \subset I_{\mathcal{C}}$. Then, for $\beta \in U_{\mathcal{C} \mathcal{L}}=f_{\mathcal{L}}^{-1}\left(I_{\mathcal{C}}\right)$ and $\beta \in U_{\mathcal{C} R}=f_{\mathcal{R}}^{-1}\left(I_{\mathcal{C}}\right)$ we obtain the existence regions for the period-2 orbits $\mathcal{O}_{\mathcal{C L}}$ and $\mathcal{O}_{\mathcal{C R}}$, respectively. As a next step we can calculate the preimages of the left interval $f_{\mathcal{L}}^{-1}\left(I_{\mathcal{C}}\right)$, obtaining the intervals $f_{\mathcal{L}}^{-2}\left(I_{\mathcal{C}}\right)$ and $f_{\mathcal{R}}^{-1} \circ f_{\mathcal{L}}^{-1}\left(I_{\mathcal{C}}\right)$.

$$
f_{\mathcal{L}}^{-2}\left(I_{\mathcal{C}}\right)=\left(\frac{1-\gamma}{8 \alpha^{2}}, \frac{1-\gamma}{4 \alpha}\right] \text { and } f_{\mathcal{R}}^{-1} \circ f_{\mathcal{L}}^{-1}\left(I_{\mathcal{C}}\right)=\left[1-\frac{1-\gamma}{4 \alpha}, 1-\frac{1-\gamma}{8 \alpha^{2}}\right)
$$

Hence, for $\beta \in U_{\mathcal{C L}^{2}}=f_{\mathcal{R}}^{-2}\left(I_{\mathcal{C}}\right)$ and $\beta \in U_{\mathcal{C R} \mathcal{L}}=f_{\mathcal{R}}^{-1} \circ f_{\mathcal{L}}^{-1}\left(I_{\mathcal{C}}\right)$, respectively, the stable period-3 orbits $\mathcal{O}_{\mathcal{C} \mathcal{L}^{2}}$ and $\mathcal{O}_{\mathcal{C R} \mathcal{L}}$ exist. By contrast, the right interval $f_{\mathcal{R}}^{-1}\left(I_{\mathcal{C}}\right)$ is for $1 / 2<\alpha<\alpha_{1}$ completely located in $(\alpha(1-\gamma), 1]$ and consequently does not have any preimages. Continuing this procedure we will see that in each step (for each $n>1)$ the left interval $f_{\mathcal{L}}^{-(n-1)}\left(I_{\mathcal{C}}\right)$ has two preimages

$$
f_{\mathcal{L}}^{-n}\left(I_{\mathcal{C}}\right)=\left(\frac{1-\gamma}{2^{n+1} \alpha^{n}}, \frac{1-\gamma}{2^{n} \alpha^{n-1}}\right] \quad \text { and } \quad f_{\mathcal{R}}^{-1} \circ f_{\mathcal{L}}^{-(n-1)}\left(I_{\mathcal{C}}\right)=\left[1-\frac{1-\gamma}{2^{n} \alpha^{n-1}}, 1-\frac{1-\gamma}{2^{n+1} \alpha^{n}}\right)
$$

whereas the right interval $f_{\mathcal{R}}^{-1} \circ f_{\mathcal{L}}^{-(n-2)}\left(I_{\mathcal{C}}\right)$ does not have any preimages. This proves that in the considered parameter range $1 / 2<\alpha<\alpha_{1}$ the period- $(n+1)$ orbits $\mathcal{O}_{\mathcal{C L}}$ and $\mathcal{O}_{\mathcal{C} \mathcal{R} \mathcal{L}^{n-1}}$ exist for all $n$. Moreover, Eq. (22) implies that for each $n$ the intervals $U_{\mathcal{C} \mathcal{L}^{n}}=f_{\mathcal{L}}^{-n}\left(I_{\mathcal{C}}\right)$ and $U_{\mathcal{C L}^{n+1}}=f_{\mathcal{L}}^{-(n+1)}\left(I_{\mathcal{C}}\right)$ are adjacent, as well as the intervals $U_{\mathcal{C} \mathcal{L} \mathcal{L}^{n-1}}=f_{\mathcal{R}}^{-1} \circ f_{\mathcal{L}}^{-(n-1)}\left(I_{\mathcal{C}}\right)$ and $U_{\mathcal{C} \mathcal{L} \mathcal{L}^{n}}=f_{\mathcal{R}}^{-1} \circ f_{\mathcal{L}}^{-n}\left(I_{\mathcal{C}}\right)$ and the union

$$
I_{\mathcal{C}} \cup\left(\bigcup_{n=1}^{\infty} f_{\mathcal{L}}^{-n}\left(I_{\mathcal{C}}\right)\right) \cup\left(\bigcup_{n=1}^{\infty} f_{\mathcal{R}}^{-1} \circ f_{\mathcal{L}}^{-(n-1)}\left(I_{\mathcal{C}}\right)\right)
$$

covers the complete interval $\beta \in(0,1)$. This proves that no other stable periodic orbits are possible in the considered range for $\alpha$. Hence, as a final result we can state that the overall set of symbolic sequences corresponding to periodic orbits existing for $1 / 2<\alpha<\alpha_{1}$ is given by

$$
L_{0}=\left\{\mathcal{C R}^{k} \mathcal{L}^{n} \mid k \in\{0,1\}, n \geq 0\right\}
$$

as illustrated in Fig. 4.

\section{Bifurcation Structure for $\alpha \leq \alpha_{\infty}$}

\subsection{Period doubling cascade of unstable orbits}

Recall that at $\alpha=\alpha^{\star}$ we observed not only stable periodic orbits corresponding to all possible symbolic sequences but also an infinite set of Milnor attractors (see $\S 1.2$ ) existing for $\beta \in M$. Therefore, it is natural to ask at which values $\alpha<\alpha^{\star}$ these Milnor attractors emerge. To clarify this question one has to recall that these attractors are given by unstable orbits of the discontinuous flat top tent map. Since these orbits contain only points located on the partitions $I_{\mathcal{L}}$ and $I_{\mathcal{R}}$, they are identical with the corresponding orbits of the usual tent map (2). Therefore, let us recall some well-known facts regarding the tent map and interpret them with respect 
to the explanation of the occurrence of unstable orbits (and hence the Milnor attractors) of the discontinuous flat top tent map.

Recall that for $\alpha<1 / 2$ the tent map has a globally attracting fixed point at the origin. At $\alpha=1 / 2$ it becomes unstable via a degenerate transcritical bifurcation [16], and an infinite number of unstable orbits emerge, namely the fixed point $\mathcal{O}_{\sigma^{1}}$ with

$$
\sigma^{1}:=\mathcal{R}
$$

as well as the periodic orbits $\mathcal{O}_{\sigma^{k}}$ with periods $2^{k}, k=1,2,3, \ldots$, and symbolic sequences

$$
\begin{aligned}
\sigma^{2} & :=\mathcal{L} \mathcal{R} \\
\sigma^{3} & :=\mathcal{R} \mathcal{R} \mathcal{L} \mathcal{R} \\
\sigma^{4} & :=\mathcal{L} \mathcal{R} \mathcal{L} \mathcal{R} \mathcal{R} \mathcal{L} \mathcal{R} \\
\sigma^{5} & :=\mathcal{R} \mathcal{R} \mathcal{L} \mathcal{R} \mathcal{R} \mathcal{L} \mathcal{R} \mathcal{L} \mathcal{R} \mathcal{L} \mathcal{R} \mathcal{R} \mathcal{L} \mathcal{R}, \\
\sigma^{6} & :=\mathcal{L} \mathcal{R} \mathcal{L} \mathcal{R} \mathcal{R} \mathcal{L} \mathcal{L} \mathcal{L} \mathcal{R} \mathcal{L} \mathcal{R} \mathcal{R} \mathcal{L} \mathcal{R} \mathcal{R} \mathcal{L} \mathcal{R} \mathcal{R} \mathcal{L} \mathcal{L} \mathcal{R} \mathcal{L} \mathcal{R} \mathcal{R} \mathcal{L} R \\
& \ldots
\end{aligned}
$$

Note that the same family of symbolic sequences describes also the orbits of the $\operatorname{logistic} \operatorname{map} x_{n+1}=\alpha x_{n}\left(1-x_{n}\right)$, forming the first period doubling cascade ${ }^{6}$. A rule how to create these sequences is described already in [12]. Alternatively, it is also possible to create these sequences starting with $\sigma^{1}:=\mathcal{R}$ and applying recursively the replacement

$$
\begin{aligned}
& \mathcal{L} \mapsto \mathcal{R} \mathcal{R} \\
& \sigma^{i} \stackrel{\mathcal{R}}{\stackrel{\mathcal{L}}{\longrightarrow}} \stackrel{\text { R }}{\longrightarrow} \sigma^{i+1}
\end{aligned}
$$

In this way, each next symbolic sequence in the family is created by replacement of each letter $\mathcal{L}($ or $\mathcal{R}$, respectively) by the syllable $\mathcal{R} \mathcal{R}(\mathcal{L} \mathcal{R})$ in the previous one, so that the overall length of the sequence is doubled in each step. The advantage of this technique is not only that it reflects the self-similarity of the underlying mechanism, but also that it allows an analytical calculation of the points of the corresponding orbits, as described in $[3]$.

Recall that in the tent map immediately after the bifurcation occurring at $\alpha=1 / 2$ all the orbits $\mathcal{O}_{\sigma^{i}}$, $i=1, \ldots, \infty$ are located close to the point $x=\frac{1}{2}$. Therefore, for the discontinuous flat top tent map all of them are virtual ${ }^{7}$ and do not (yet) exist as long as $\alpha$ is (still) close to $1 / 2$. However, with increasing $\alpha$ the orbits grow and move away from $x=1 / 2$ becoming, one after another, real (non-virtual). As an example, Fig. 7 shows the first three unstable orbits from the sequence mentioned above: the fixed point $\mathcal{O}_{\sigma^{1}}$, the period-2 orbit $\mathcal{O}_{\sigma^{2}}$ and the period- 4 orbit $\mathcal{O}_{\sigma^{3}}$. As one can see, the orbits emerge at $\alpha=1 / 2$ at the boundary $x=1 / 2$ and leave the middle interval $((1-\gamma) / 2,(1+\gamma) / 2)$ at the parameter values $\alpha_{1}, \alpha_{2}, \alpha_{3}$, respectively. At these parameter values they become also real orbits of the discontinuous flat top tent map. Therefore, the discontinuous flat top tent map shows some remarkable similarity not only with the tent map but also with the logistic map:

- Similar to both the logistic and the tent map, the discontinuous flat top tent map shows a sequence of orbits corresponding to the sequences $\sigma^{i}, i=1, \ldots, \infty$.

\footnotetext{
${ }^{6}$ When dealing with the logistic map, the application of symbolic dynamics for the description of an orbit requires some more comments. One possible way used by many researchers is to define the partitions associated with the letters $\mathcal{L}$ and $\mathcal{R}$ on the left and on the right of the maximum point $x=1 / 2$. In this case the problem arises that the symbolic description of an orbit changes at the parameter value for which the orbit contains the point $x=1 / 2$. A possible solution of this problem is to define the symbolic sequence associated with an orbit after this crossing of the partition boundary, or generally for $\alpha \geq 4$. The sequences given in Eq. (25) are defined in this way.

${ }^{7}$ Recall that when dealing with piecewise-smooth systems it is common to denote an orbit as virtual if it contains points located in a "wrong" partition, that means a partition where the corresponding function is not applicable.
} 


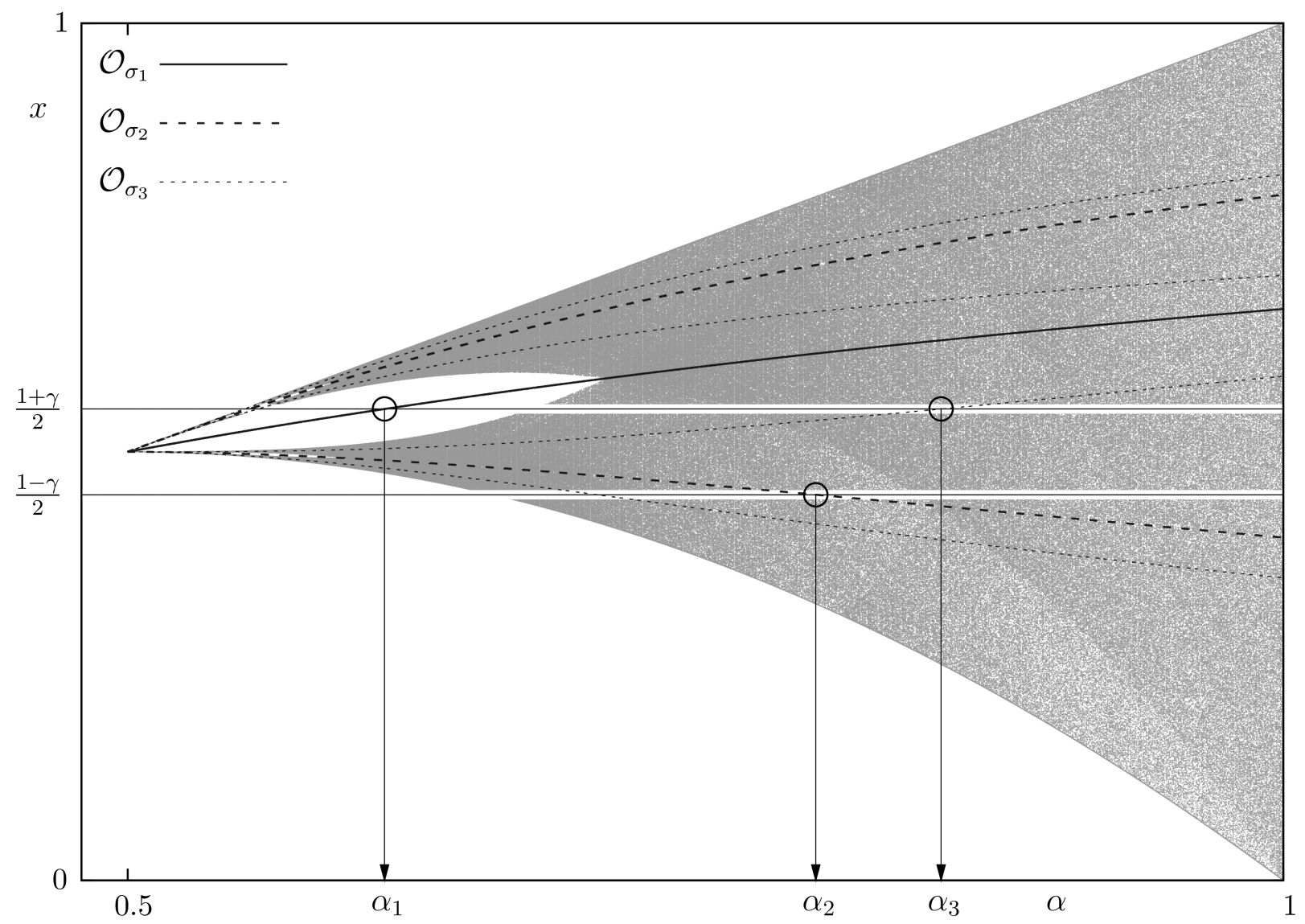

FiguRE 7. Bifurcation diagram of the usual tent map (2) and the unstable orbits $\mathcal{O}_{\sigma^{1}}$ (fixed point), $\mathcal{O}_{\sigma^{2}}$ (period 2), $\mathcal{O}_{\sigma^{3}}$ (period 4). As long as some points of an orbit are located in the interval $[(1-\gamma) / 2,(1+\gamma) / 2]$, the orbit is virtual for the discontinuous flat top tent map. The parameter values $\alpha_{i}, i=1,2,3$ are shown, where the orbits $\mathcal{O}_{\sigma^{i}}$ appear in the discontinuous flat top tent map.

- Similar to the logistic map (and by contrast to the tent map) these orbits emerge via a sequence of bifurcations and not via one bifurcation. As in the case of a period doubling cascade, for each $i$, the orbit corresponding to the sequence $\sigma^{i}$ emerges at the parameter value $\alpha_{i}<\alpha_{i+1}$, and the sequence of these bifurcations converges to a value $\alpha_{\infty}$. However, by contrast to the logistic map, the cascade is formed not by flip bifurcations but by border collision bifurcations.

- Similar to the tent map (and by contrast to the logistic map) these orbits are everywhere unstable.

Note also that since the orbits $\mathcal{O}_{\sigma^{i}}, i=1, \ldots, \infty$ are located on the partitions $I_{\mathcal{L}}$ and $I_{\mathcal{R}}$ only (as any other unstable periodic orbit of the discontinuous flat top tent map), their existence regions $\mathcal{P}_{\sigma^{i}}$ do not depend on $\beta$ :

$$
\mathcal{P}_{\sigma^{i}}=\left\{(\alpha, \beta) \mid \alpha>\alpha_{i}\right\}
$$

\subsection{Fixed point $\mathcal{O}_{\sigma^{1}}$ as a Milnor attractor}

Let us consider first the situation close to $\alpha=\alpha_{1}$ (see Eq. (19)), where the fixed point $\mathcal{O}_{\sigma^{1}}$ reaches the partition $I_{\mathcal{R}}$ and becomes real. Then, by setting the value $\beta$ to this unstable fixed point or to one of its 
preimages one guarantees that a typical initial value $x_{0} \in \widetilde{S}$ after reaching the middle partition $I_{\mathcal{C}}$ is mapped either directly or after some number of steps to $x_{\sigma^{1}}$, and hence for these values of $\beta$ the unstable fixed point $\mathcal{O}_{\sigma^{1}}$ represents a Milnor attractor ${ }^{8}$. An example for this situation is shown in Fig. 8. As one can see in this figure, a trajectory started at an arbitrary chosen initial value $x_{0} \neq x_{\sigma^{1}}$ reaches after some number of iterations the middle partition $I_{\mathcal{C}}$. After that the particular choice of the initial value does not play any role and the remaining part of the trajectory is determined by the value of $\beta$ only. In the presented example this value is set to one of the rank-5 preimages of the unstable fixed point $\mathcal{O}_{\sigma^{1}}$ and hence for any initial value the trajectory will be mapped onto this fixed point in five steps after the point located in the middle partition, that means $x_{k} \in I_{\mathcal{C}}$ implies $x_{k+5}=x_{\sigma^{1}}$.

For a given value of $\alpha$, the corresponding set of $\beta$ values is denoted in the following as

$$
B_{\sigma^{1}}(\alpha):=\left\{\beta \mid \exists k \geq 0: f^{k}(\beta)=x_{\sigma^{1}}\right\}
$$

For a fixed $\alpha$, the set $B_{\sigma^{1}}$ is a set of points, and in the $(\alpha, \beta)$ parameter plane it forms a set of curves, as shown in Fig. 9. As one can see, these curves represent the limiting values of the nested period incrementing cascades (for example, of all nested cascades in the scenario shown in Fig. 2b). In order to explain that, we need to extend our notation. Let us introduce first the set of the values of $\beta$ which are mapped to $\mathcal{O}_{\sigma^{1}}$ in exact $k$ steps:

$$
B_{\sigma^{1}}^{k}(\alpha):=\left\{\beta \mid f^{k}(\beta)=x_{\sigma^{1}}, \text { and } \nexists j<k: f^{j}(\beta)=x_{\sigma^{1}}\right\}
$$

Obviously, the set $B_{\sigma^{1}}(\alpha)$ introduced before is simply given by $B_{\sigma^{1}}(\alpha)=\bigcup_{k=0}^{\infty} B_{\sigma^{1}}^{k}(\alpha)$. It is clear that for any value of $\alpha>\alpha_{1}$ the set $B_{\sigma^{1}}^{0}(\alpha)$ contains only one point $\beta=x_{\sigma^{1}}$. Similarly, $B_{\sigma^{1}}^{1}(\alpha)$ contains also only one point $\beta=f_{\mathcal{L}}^{-1}\left(x_{\sigma^{1}}\right)=1 / 2-x_{\sigma^{1}}$ (the only rank-1 preimage of $\left.x_{\sigma^{1}}\right)$. By contrast, already the set $B_{\sigma^{1}}^{2}$ contains more values, namely the two rank-2 preimages of $\mathcal{O}_{\sigma^{1}}$, given by $\beta=f_{\mathcal{L}}^{-1} \circ f_{\mathcal{L}}^{-1}\left(x_{\sigma^{1}}\right)$ and $\beta=f_{\mathcal{R}}^{-1} \circ f_{\mathcal{L}}^{-1}\left(x_{\sigma^{1}}\right)$. For the next set, $B_{\sigma^{1}}^{3}(\alpha)$, we can state that close to $\alpha_{1}$ it contains only two values $\beta=f_{\mathcal{L}}^{-1} \circ f_{\mathcal{L}}^{-1} \circ f_{\mathcal{L}}^{-1}\left(x_{\sigma^{1}}\right)$ and $\beta=f_{\mathcal{R}}^{-1} \circ f_{\mathcal{L}}^{-1} \circ f_{\mathcal{L}}^{-1}\left(x_{\sigma^{1}}\right)$ but for increasing values of $\alpha$ further preimages appear and enlarge the number of elements in this set. In order to distinguish between these preimages (and to have a unique notation for each curve in the $(\alpha, \beta)$-plane), let us specify the path $\varrho=\varrho_{0} \varrho_{1} \ldots \varrho_{k-1}$ of minimal length $k$ leading from the middle partition to $\mathcal{O}_{\sigma^{1}}$ :

$$
\begin{gathered}
B_{\sigma^{1}}^{\varrho}(\alpha):=\left\{\beta \mid f_{\varrho_{k-1}} \circ f_{\varrho_{k-2}} \circ \cdots \circ f_{\varrho_{0}}(\beta)=x_{\sigma^{1}} \text { and } \nexists j<k: f_{\varrho_{j-1}} \circ f_{\varrho_{j-2}} \circ \cdots \circ f_{\varrho_{0}}(\beta)=x_{\sigma^{1}},\right. \\
\left.\varrho_{i} \in\{\mathcal{L}, \mathcal{R}\}, i=0 . . k-1\right\}
\end{gathered}
$$

In the examples above we have $B_{\sigma^{1}}^{0}(\alpha)=B_{\sigma^{1}}^{\varepsilon}(\alpha)=x_{\sigma^{1}}, B_{\sigma^{1}}^{1}(\alpha)=B_{\sigma^{1}}^{\mathcal{L}}(\alpha), B_{\sigma^{1}}^{2}(\alpha)=B_{\sigma^{1}}^{\mathcal{L}}(\alpha) \cup B_{\sigma^{1}}^{\mathcal{R}}(\alpha)$, and so on. Moreover (see also Fig. 9), close to $\alpha_{1}$ we can easily demonstrate that for any $k>1$ the fixed point $\mathcal{O}_{\sigma^{1}}$ has exactly two rank- $k$ preimages:

$$
B_{\sigma^{1}}^{k}(\alpha)=B_{\sigma^{1}}^{\mathcal{L}^{k}}(\alpha) \cup B_{\sigma^{1}}^{\mathcal{R} \mathcal{L}^{k-1}}(\alpha)
$$

Indeed, the fixed point $\mathcal{O}_{\sigma^{1}}$ has an infinite sequence of preimages on the left side, accumulating to the unstable fixed point $\mathcal{O}_{\mathcal{L}}$. Each of these preimages has additionally one preimage on the right side, which however, do not have any further preimages, as shown in $\S 1.4$.

All other paths from the middle partition to the unstable fixed point emerge at homoclinic bifurcations which occur for larger values of $\alpha$ (see $\S 2.4$ for details). In Fig. 9 the curves corresponding to paths of length $k \leq 7$ are marked. As one can see, the number of these curves increases for $\alpha \rightarrow \alpha^{\star}$. Moreover, at the value $\alpha=\alpha^{\star}$ we can state that every symbolic sequence which ends with $\mathcal{L}$ represents a possible path $\varrho=\varrho_{0} \varrho_{1} \ldots \varrho_{k-1}$ from the middle partition to the unstable fixed point:

$$
B_{\sigma^{1}}^{k}\left(\alpha^{\star}\right)=\left\{B_{\sigma^{1}}^{\varrho}\left(\alpha^{\star}\right) \mid \varrho_{i} \in\{\mathcal{L}, \mathcal{R}\}, \quad i=0 . . k-2, \varrho_{k-1}=\mathcal{L}\right\}
$$

\footnotetext{
${ }^{8}$ Note that in the following we use the notation $\mathcal{O}_{\sigma^{1}}$ for the fixed point and the notation $x_{\sigma^{1}}$ for its value. This may look unnecessary and misleading, since clearly $\mathcal{O}_{\sigma^{1}}=\left\{x_{\sigma^{1}}\right\}$. The notation is used for unification of the expressions for any periods, since for the period-2 orbit we have $\mathcal{O}_{\sigma^{2}}=\left\{x_{\sigma^{2}}^{0}, x_{\sigma^{2}}^{1}\right\}$, for the period-4 orbit we have $\mathcal{O}_{\sigma^{3}}=\left\{x_{\sigma^{3}}^{0}, \ldots, x_{\sigma^{3}}^{3}\right\}$, and so on.
} 

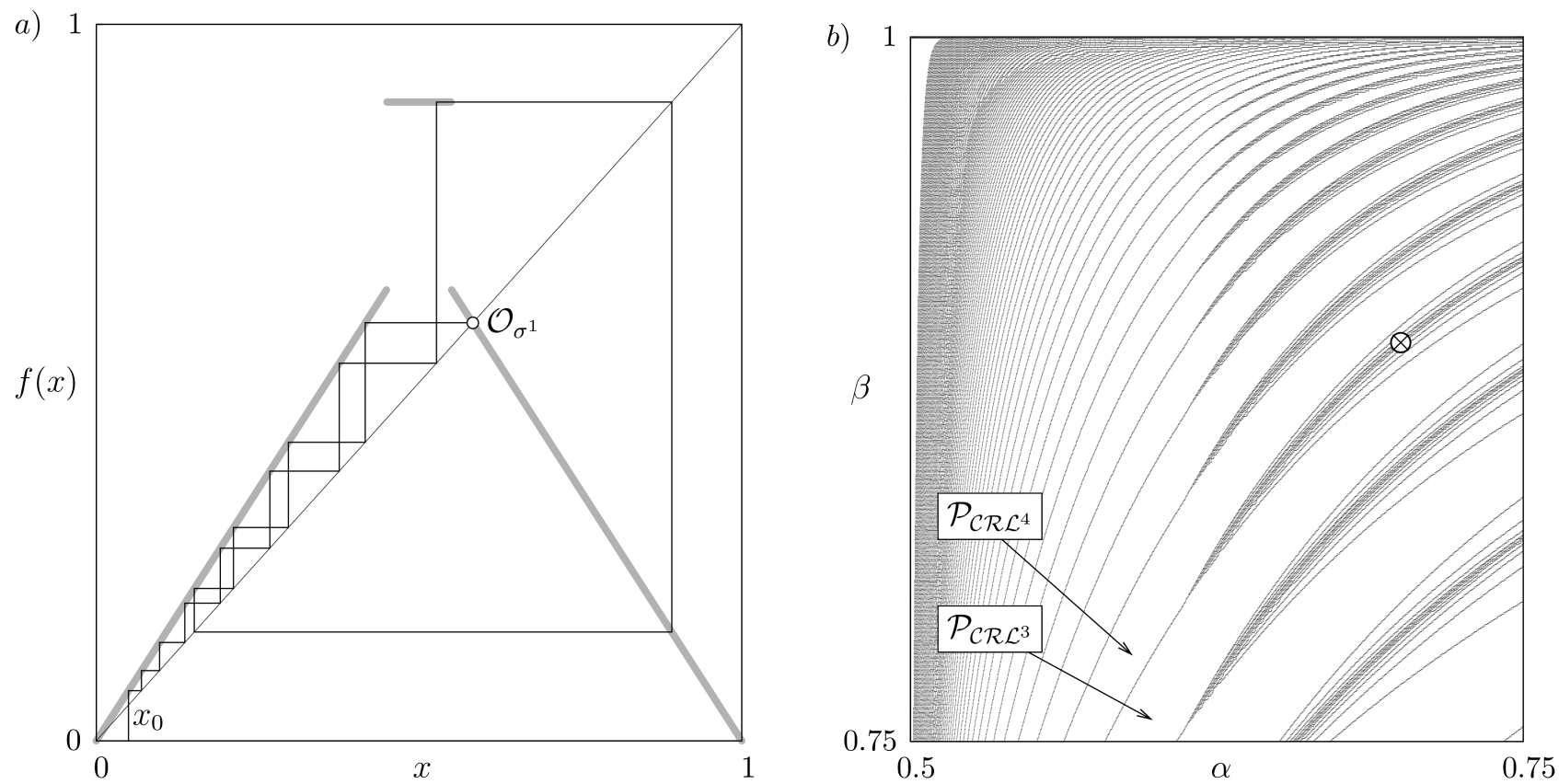

FiguRE 8. a) A trajectory at $a=0.7, \beta=B_{\sigma^{1}}^{\mathcal{R} \mathcal{L}^{4}}(\alpha) \approx 0.89152$ converges to the unstable fixed point $\mathcal{O}_{\sigma^{1}}$ which represents in this case a Milnor attractor. In b) this point in the parameter plane $(\alpha, \beta)$ is marked with a cross.

with $k>1$. The fact that the last symbol in the sequence $\varrho$ must be an $\mathcal{L}$ is easily explained, since it correspond to the rank-1 preimage of the fixed point, and the only rank-1 preimage of the fixed point is located in the left partition. All other symbols may be both $\mathcal{L}$ and $\mathcal{R}$, since for $\alpha=\alpha^{\star}$ the discontinuous flat top tent map is surjective onto $[0,1]$, and therefore any preimage of the fixed point $\mathcal{O}_{\mathcal{R}}$ has both a preimage in $I_{\mathcal{L}}$ and a preimage in $I_{\mathcal{R}}$.

Now we can begin establishing the connection between stable and unstable orbits of the discontinuous flat top tent map existing at a particular value $\alpha<\alpha_{\infty}$. As we have shown in the previous section, all orbits corresponding to the sequences from the set $L_{0}$ (as defined in $\S 1.4$ ) persist for $\alpha \geq \alpha_{1}$, but additionally an infinite number of nested cascades emerges and the set of symbolic sequences corresponding to the orbits forming these cascades is given by

$$
L_{1}=\left\{\varrho \mathcal{R}^{n} \mid \varrho \in L_{0}, n>0\right\} .
$$

More precisely, the situation around the value $\alpha_{1}$ can be described as follows. For $\alpha<\alpha_{1}$ there exist only orbits corresponding to sequences $\varrho \in L_{0}$. After the unstable fixed point $\mathcal{O}_{\sigma^{1}}$ appears at $\alpha_{1}$, each region corresponding to an orbit with a symbolic sequence $\varrho \in L_{0}$ becomes augmented to one side by two cascades formed by the orbits with the symbolic sequence

$$
\left\{\varrho\left(\sigma^{1}\right)^{2 n} \mid n>0\right\} \text { and }\left\{\varrho\left(\sigma^{1}\right)^{2 n-1} \mid n>0\right\} .
$$

For $n$ going to infinity each of these cascades converge to the parameter value $B_{\sigma^{1}}^{\varrho}$. The mechanism causing these cascades to emerge is explained below, in $\S 2.3$.

Schematically this structure is shown in Fig. 10. For $\alpha<\tilde{\alpha}$ the existence regions $\mathcal{P}_{\varrho}$ and $\mathcal{P}_{\varrho^{\prime}}$ of the stable orbits $\mathcal{O}_{\varrho}$ and $\mathcal{O}_{\varrho^{\prime}}$ (shown in different shades of gray) are adjacent in parameter space. At $\alpha=\tilde{\alpha}$ the unstable orbit $\mathcal{O}_{\tilde{\sigma}}$ emerges (in the case $\tilde{\sigma}=\sigma^{1}$ we have of course $\tilde{\alpha}=\alpha_{1}$, but the situation is structurally the same 


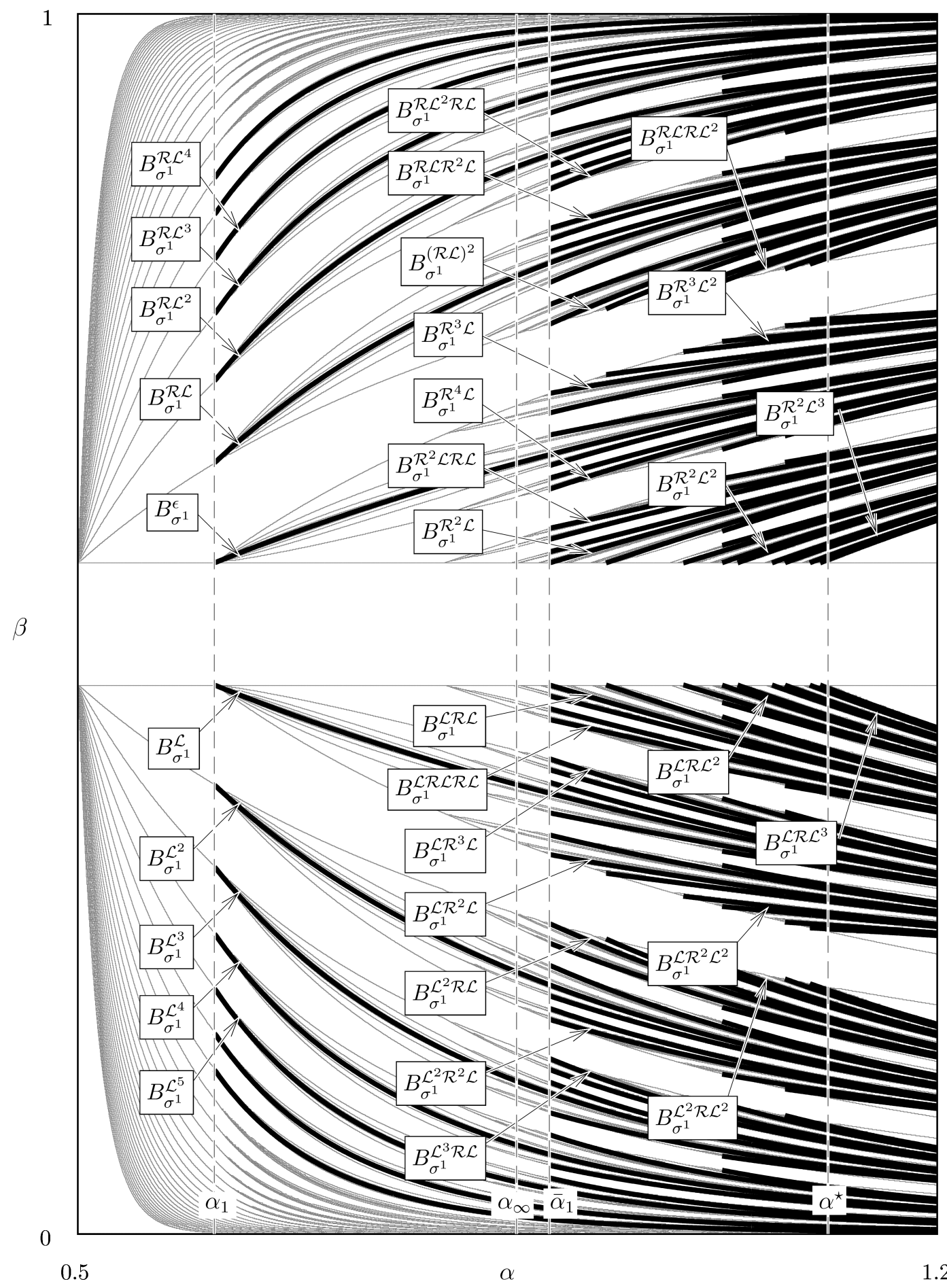

Figure 9. Black curves show the sets $B_{\sigma^{1}}^{k}$ with $k \leq 7$ of parameter values where the unstable fixed point $\mathcal{O}_{\sigma^{1}}$ represents a Milnor attractor. Labeled are the curves from the sets $B_{\sigma^{1}}^{k}$ with $k \leq 5$. The value $\bar{\alpha}_{1}$ refers to a homoclinic bifurcation after which a non-critical homoclinic orbit to the unstable fixed point emerges (see $\S 2.4$ ). 


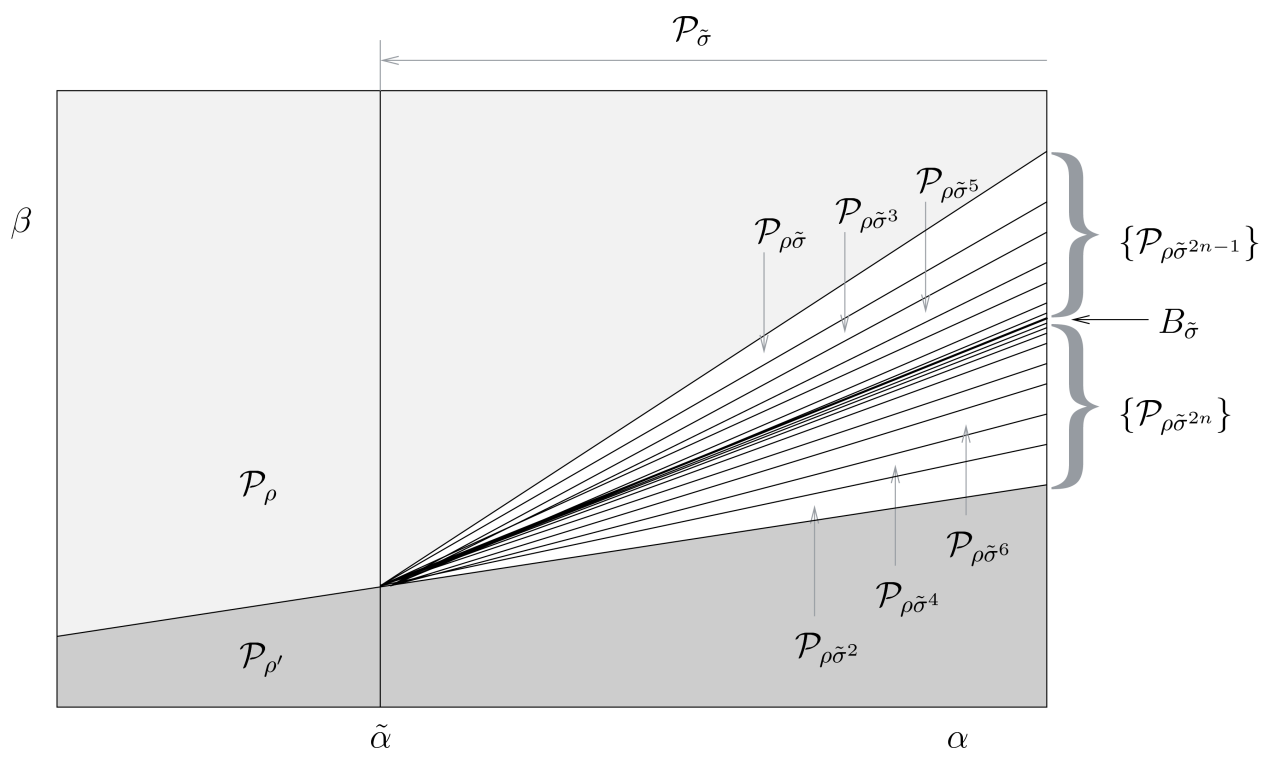

FIgURE 10. Schematic representation of the bifurcation structure close to the value $\tilde{\alpha}$ where the unstable periodic orbit $\mathcal{O}_{\tilde{\sigma}}$ emerges. For details see text.

for any other $\tilde{\sigma}$ as well) and the regions $\mathcal{P}_{\varrho}$ and $\mathcal{P}_{\varrho^{\prime}}$ become separated by two period incrementing cascades converging to the curve in the parameter space where the unstable orbit $\mathcal{O}_{\tilde{\sigma}}$ represents a Milnor attractor.

Note also that we can compare now the nested period incrementing scenario with the well-known period adding scenario, where the periods of stable orbits are organized in the way that between the existence regions of orbits with the periods $p_{1}$ and $p_{2}$ there exists a region with the period $p_{1}+p_{2}$, and the rotation numbers of all periodic orbits form the well-known Farey-tree. In fact, comparing the bifurcation structures in both cases (especially in 2D parameter spaces), one can see that they are amazingly similar. Both scenarios are self-similar and have an infinite number of accumulation points. However, there exists a significant difference between both scenarios (apart the trivial one, namely that the rules how the periodicity regions are located with respect to each other are different). Indeed, in the period-adding scenario the behavior at the accumulation points is known to be quasi-periodic. This seems to be quite natural, as a sequence of increasing periods converges to the "infinite period" in this case. In the case of the discontinuous flat top tent map the situation is different. At the accumulation point the behavior is given not by a quasi-periodic trajectory but by a Milnor attractor which can be given by any unstable periodic orbit. In the case we considered above (close to $\alpha_{1}$ ) this Milnor attractor is given by a fixed point so that the cascades of increasing periods converge to a value where the period of the attractor is one.

\subsection{Connection between stable and unstable orbits}

Let us now reconsider the situation before and after the bifurcation line $\alpha=\alpha_{1}$ in the $(\alpha, \beta)$ parameter plane from a more general perspective. As we have seen, there are three facts which we have to connect to each other:

(1) Before the bifurcation line there exists a set of stable periodic orbits. In the case we considered so far, the symbolic sequences of these orbits are $\varrho \in L_{0}$.

(2) At the bifurcation line an unstable orbit with the symbolic sequence $\sigma$ emerges. In the case we considered so far, this unstable orbit is the fixed point $\mathcal{O}_{\sigma^{1}}$.

(3) After the bifurcation line a set of stable periodic orbits exists, whereby the symbolic sequences of the orbits emerging at the bifurcation point can be written as $L_{1}=\left\{\varrho \sigma^{n}, n \geq 1\right\}$. 

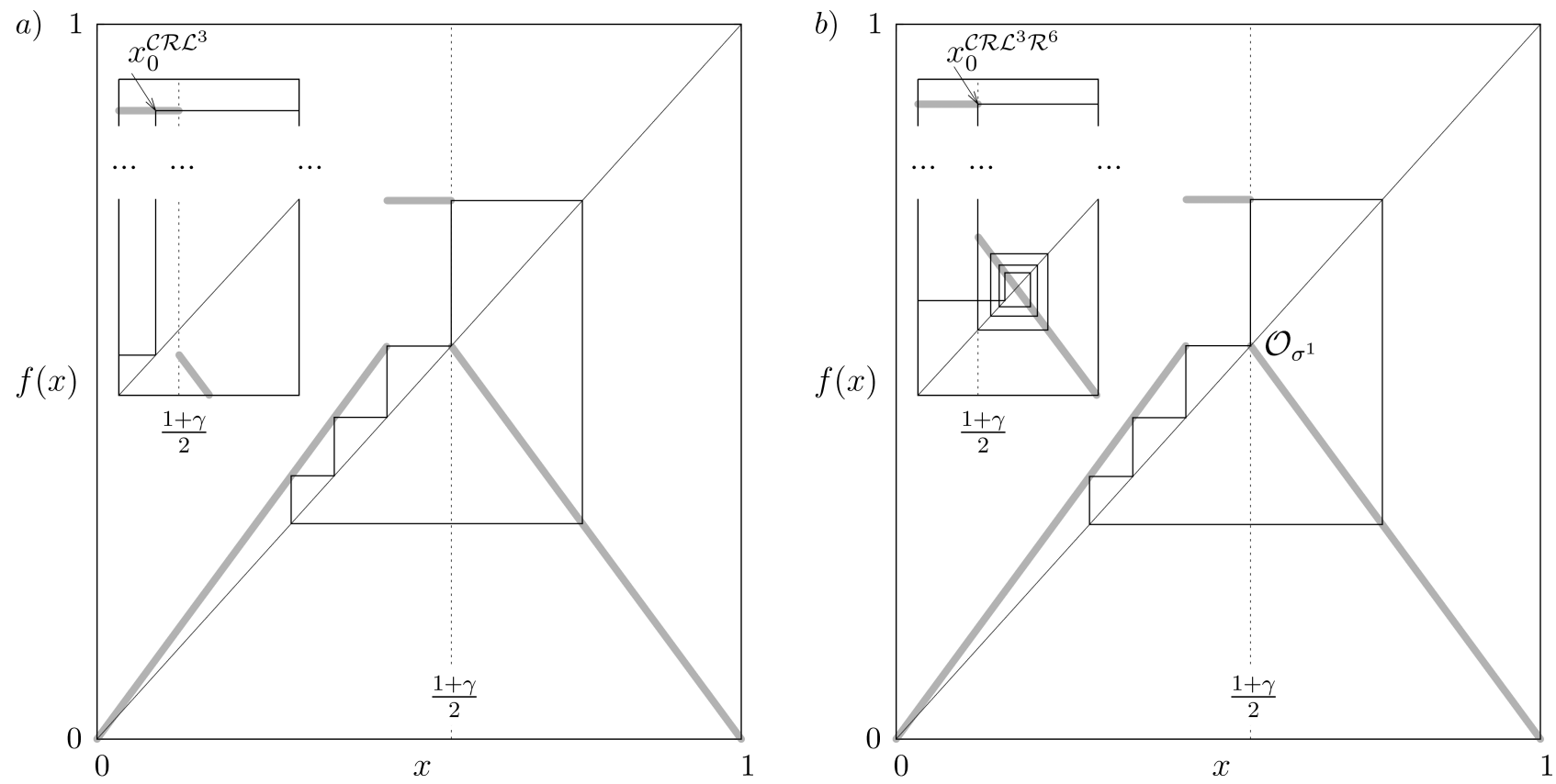

FiguRE 11. Stable periodic orbits close before and close after the bifurcation point $\alpha=\alpha_{1}$. The insets show the situations in a small interval (of the size $1.5 \cdot 10^{-3}$ ) close to the right boundary of the middle partition. In (a), at $\alpha=0.61<\alpha_{1} \beta=0.7547$, there is the stable orbit $\mathcal{O}_{\varrho}$ with $\varrho=\mathcal{C} \mathcal{R} \mathcal{L}^{3}$, whereas the unstable fixed point $\mathcal{O}_{\sigma^{1}}$ is still virtual. In (b), at $\alpha=0.6119>\alpha_{1}, \beta=0.753277$, the unstable fixed point $\mathcal{O}_{\sigma^{1}}$ exists, and the stable orbit is $\mathcal{O}_{\varrho\left(\sigma^{1}\right)^{6}} \equiv \mathcal{O}_{\mathcal{C} \mathcal{R} \mathcal{L}^{3} \mathcal{R}^{6}}$

In other words, the symbolic sequences of the stable orbits emerging at the bifurcation point can be created by taking all symbolic sequences of the stable orbits existing before the bifurcation and appending to each of them a suffix defined by the symbolic sequence of the unstable orbit emerging at the bifurcation point, repeated an arbitrary number of times. In the following we will demonstrate that the described situation occurs not only at the point $\alpha_{1}$ but at any bifurcation value where an unstable orbit appears. Moreover, the same situation happens not only when a "new" unstable orbit appears, but also when an already existing unstable orbit undergoes a homoclinic bifurcation. At these bifurcations (see for an example the point $\alpha=\bar{\alpha}_{1}$ in Fig. 9) new preimages of an already existing unstable orbit appear, and also new incrementing cascades converging to the Milnor attractor given by the "old" unstable orbit. In the present work we are not demonstrating the complete proof in all details, but restrict ourselves to the basic idea of the proof only.

Consider some stable periodic orbit $\mathcal{O}_{\varrho}$ at a fixed value $\alpha:=\alpha_{1}^{-}=\alpha_{1}-\varepsilon$, slightly before the bifurcation. The interval $\left[\beta_{\min }^{\varrho}, \beta_{\max }^{\varrho}\right]$ of the values of $\beta$ for which this orbit exists is bounded by two border collision bifurcations. For simplicity, let us further exclude from consideration the only stable orbit $\mathcal{O}_{\mathcal{C R}}$ which does not contain any point in the left partition. The final results for this orbit will be the same, but the reasoning is slightly different. Under this assumption, we can state that the last symbol in $\varrho$ is $\mathcal{L}$.

Consider now the situation when an unstable orbit $\mathcal{O}_{\sigma}$ of the discontinuous flat top tent map emerges. As already mentioned, all these orbits consist of points located on the left and on the right partitions only, and hence are identical with the orbits of the usual tent map with the additional condition that all points of the orbit have already left the interval $[(1-\gamma) / 2,(1+\gamma) / 2]$. For the tent map we know that any unstable orbit (in fact: any periodic orbit except the unstable fixed point $\mathcal{O}_{\mathcal{L}}$ ) emerges with all points located close to the boundary $x=1 / 2$. Then, for increasing $\alpha$, the points move away from $x=1 / 2$ and leave - one after another 
a)

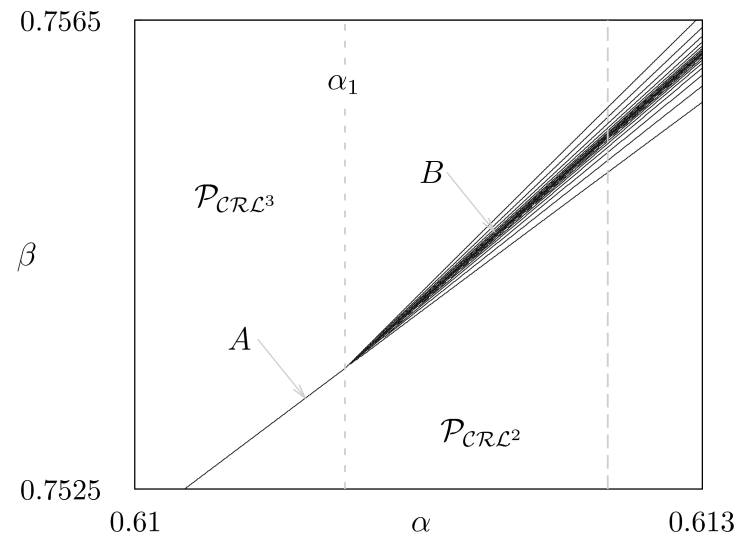

c)

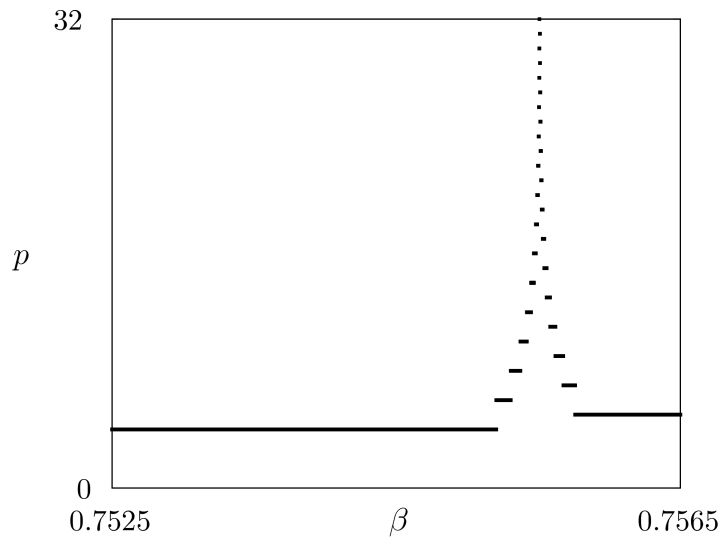

b)

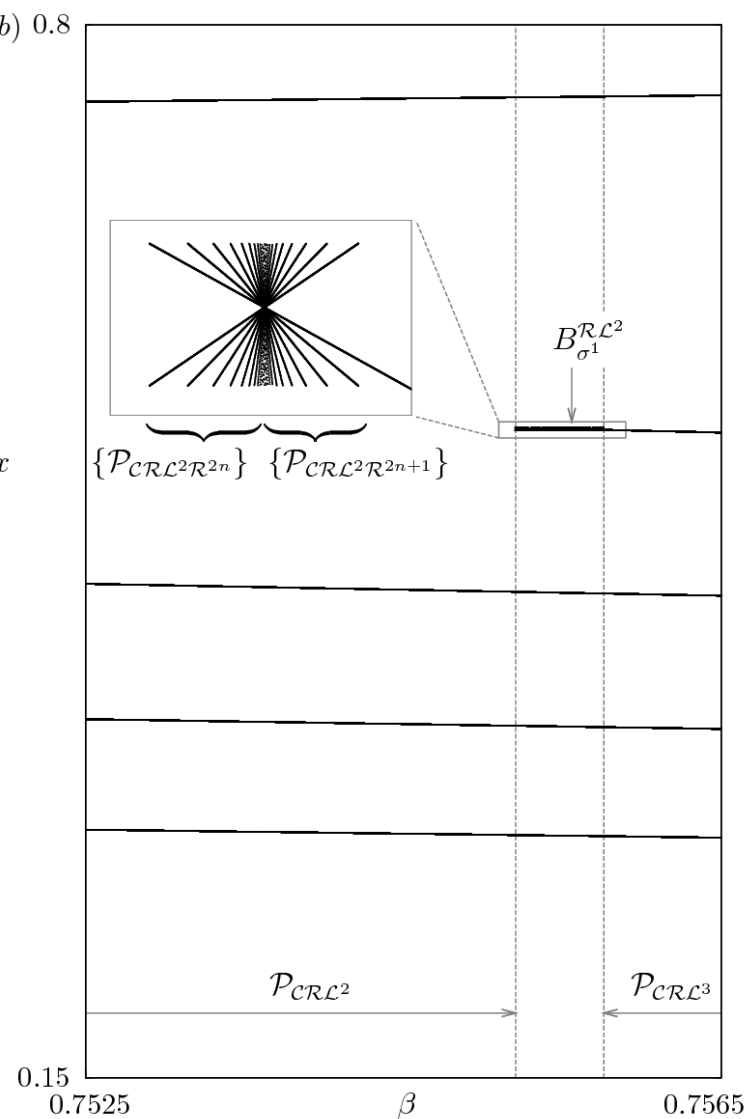

FiguRE 12. In a) the bifurcation structure in the $(\alpha, \beta)$ parameter plane is shown close to the bifurcation line $\alpha=\alpha_{1}$ where the unstable fixed point $\mathcal{O}_{\sigma^{1}}$ emerges (cmp. schematic representation in Fig. 10). The points marked with $A$ and $B$ correspond to Figs.11 $a$ and $11 b$, respectively. In (b) and (c) the bifurcation and the period diagram, respectively, are shown, for the $1 \mathrm{D}$ bifurcation scenario occurring at $\alpha=0.6125$, along the vertical dashed line marked in a). The inset in the bifurcation diagram (b) shows the marked rectangle enlarged.

- the interval $[(1-\gamma) / 2,(1+\gamma) / 2]$ (see Fig. 7 for the first three orbits in the sequence $\left\{\mathcal{O}_{\sigma^{i}} \mid i=1,2,3, \ldots\right\}$ ). As soon as the last point of the orbit $\mathcal{O}_{\sigma}$ has left this interval, the orbit becomes real (non-virtual) for the discontinuous flat top tent map. Therefore we can state that at the bifurcation point where an unstable orbit of the discontinuous flat top tent map emerges, it has a point either at $(1-\gamma) / 2$ or at $(1+\gamma) / 2$ (for the orbits in the sequence $\left\{\mathcal{O}_{\sigma^{i}} \mid i=1,2,3, \ldots\right\}$ one can easily see that the orbits with odd $i$ emerge with a point located at the right boundary $(1+\gamma) / 2$, and the orbits with even $i$ emerge with a point located at the left boundary $(1-\gamma) / 2)$.

Fig. $11 a$ illustrates the situation for $\alpha=\alpha_{1}^{-}$. As one can see in the inset showing the situation close to the right boundary of the middle partition, the unstable fixed point $\mathcal{O}_{\sigma^{1}}$ is still not emerged, and the attractor is given by the stable orbit $\mathcal{O}_{\varrho}$ with $\varrho=\mathcal{C} \mathcal{R} \mathcal{L}^{3}$. Let us denote the point of this orbit located in the middle partition by $x_{0}^{\varrho}$ and then let us define the iterated function

$$
f_{\varrho}(x):=f_{\varrho_{n}} \circ f_{\varrho_{n-1}} \circ \cdots \circ f_{\varrho_{1}}(\underbrace{f_{\mathcal{C}}(x)}_{\beta})
$$


Clearly, for $\alpha<\alpha_{1}$ the function $f_{\varrho}$ maps the point $x_{0}^{\varrho}$ onto itself. However, after the bifurcation the situation changes. Therefore, let us consider the situation for a value $\alpha=\alpha_{1}^{+}:=\alpha_{1}+\varepsilon$ slightly above the bifurcation point $\alpha_{1}$. As shown in Fig. $11 b$, the fixed point $\mathcal{O}_{\sigma^{1}}$ exists already, and there exists also a small interval between this fixed point and the partition boundary $\frac{1+\gamma}{2}$.

Let us now consider again the effect of the function $f_{\varrho}$ on a point located in the middle partition close to its boundary. Clearly, the fact that an unstable fixed point $\mathcal{O}_{\sigma^{1}}$ has appeared does not influence directly the existence of the stable orbit $\mathcal{O}_{\varrho}$ : Changing $\alpha$ from $\alpha_{1}^{-}$to $\alpha_{1}^{+}$we observe that the interval $\left[\beta_{\min }^{\varrho}, \beta_{\max }^{\varrho}\right]$ is only slightly shifted. Hence, for $\alpha_{1}^{+}$we can slightly change $\beta$ so that the situation remains unchanged: the function $f_{\varrho}$ still maps the point $x_{0}^{\varrho}$ onto itself. However, when changing $\beta$ further, across the boundary of the interval $\left[\beta_{\min }^{\varrho}, \beta_{\max }^{\varrho}\right]$, the situation changes: The point $x_{0}^{\varrho}$ will be mapped by $f_{\varrho}$ on the opposite side of the partition boundary $(1+\gamma) / 2$, that means on some point in the interval between $(1+\gamma) / 2$ and the unstable fixed point $\mathcal{O}_{\sigma^{1}}$ mentioned above. Moreover, varying $\beta$ we can put the point $f_{\varrho}\left(x_{0}^{\varrho}\right)$ arbitrarily close to this fixed point or directly onto it. Clearly, in the last case we have $f_{\varrho}\left(x_{0}^{\varrho}\right)=x_{\sigma^{1}}$ and the fixed point becomes a Milnor attractor. By contrast, in the first case the orbit will perform some number of rotations around the fixed point $\mathcal{O}_{\sigma^{1}}$. As the fixed point is unstable, the orbit will rotate to the outside and will necessarily reach the middle partition where the orbit becomes periodic. Furthermore, the orbit is locally confined to the neighborhood of $\mathcal{O}_{\sigma^{1}}$, as $f_{\mathcal{R}}^{2}((1+\gamma) / 2)>(1-\gamma) / 2$ at $\alpha=\alpha_{1}^{+}$, which means that the orbit cannot go from the swirl directly to the left partition $^{9}$. The symbolic sequence corresponding to this stable periodic orbit is $\varrho\left(\sigma_{1}\right)^{n}$ with an even number $n$, since each rotation around $\mathcal{O}_{\sigma^{1}}$ means two iteration steps. It is easy to see that starting the rotations arbitrarily close to $\mathcal{O}_{\sigma^{1}}$ an arbitrary number of rotations can be achieved, so that all the orbits with the point $f_{\varrho}\left(x_{0}^{\varrho}\right)$ located in the interval between $(1+\gamma) / 2$ and the unstable fixed point $\mathcal{O}_{\sigma^{1}}$ form the period incrementing cascade with the increment value two (corresponding to a complete additional rotation). Moreover, when the orbit is injected into the swirl on the other side of $\mathcal{O}_{\sigma^{1}}$, the effect will be the same, except that the symbolic sequences of the resulting periodic orbits are $\varrho\left(\sigma_{1}\right)^{n}$ with any odd number $n$, i.e., performing an extra half rotation.

Note that for any $n$ the bifurcation curves bounding the existing regions of the orbits $\mathcal{O}_{\varrho\left(\sigma_{1}\right)^{n}}$ forming both cascades with even and with odd $n$ converge to a point at the line $\alpha=\alpha_{1}$ (see for an example Fig.12a). This can be easily seen because for $\alpha$ approaching $\alpha_{1}$ from the right side the interval between the partition boundary $(1+\gamma) / 2$ and the unstable fixed point $\mathcal{O}_{\sigma^{1}}$ shrinks to zero ${ }^{10}$. Therefore, at the line $\alpha=\alpha_{1}$ there exist an infinite number (one for every $\varrho$ ) of codimension-2 points (big bang bifurcation points) where an infinite number of border collision bifurcation curves are issuing from.

The described situation is illustrated in Fig.12. As one can see in Fig.12a, for $\alpha<\alpha_{1}$ there exist only the orbits $\mathcal{O}_{\mathcal{C} \mathcal{R} \mathcal{L}^{2}}$ and $\mathcal{O}_{\mathcal{C} \mathcal{R} \mathcal{L}^{3}}$ in the considered region of the parameter space. At $\alpha=\alpha_{1}$ the complexity of the bifurcation structure increases and two period incrementing cascades appear, both converging to the value $\beta=B_{\sigma^{1}}^{\mathcal{C} \mathcal{L} \mathcal{L}^{2}}$. As one can see in the bifurcation diagram shown in Fig. $12 b$, the cascade located more close to the region $\mathcal{P}_{\mathcal{C} \mathcal{R} \mathcal{L}^{2}}$ is formed by the orbits corresponding to the symbolic sequences $\mathcal{C} \mathcal{R} \mathcal{L}^{2} \mathcal{R}^{2 n}$, and the other cascade by the orbits corresponding to the symbolic sequences $\mathcal{C} \mathcal{R} \mathcal{L}^{2} \mathcal{R}^{2 n+1}$.

It is worth to note that the same reasoning can be applied not only for the fixed point $\mathcal{O}_{\sigma^{1}}$ but for any other unstable periodic orbit. Of course, if an unstable orbit emerges with a point on the left boundary $(1-\gamma) / 2$, as for example the period-2 orbit $\mathcal{O}_{\sigma^{2}}$, we have to consider the situation close to that boundary. In this case, also the location of the even/odd cascades with respect to $B_{\sigma}^{\varrho}$ is switched. This is also the case (independently of $\sigma)$ each time $\varrho$ has odd $\mathcal{R}$-parity.

Nevertheless, we can state as a final result that the appearance of any unstable periodic orbit $\mathcal{O}_{\sigma}$ at some value $\alpha=\tilde{\alpha}$ implies also the appearance of an infinite number of stable periodic orbits $\mathcal{O}_{\varrho \sigma^{n}}$ for any $n$ and for any $\varrho$ for which the stable orbit $\mathcal{O}_{\varrho}$ exists for $\alpha<\tilde{\alpha}$. For each fixed $\varrho$ these orbits are organized in two period incrementing cascades (one with odd and one with even $n$ ), converging to the same value of $\beta$. At this value

\footnotetext{
${ }^{9}$ This will be possible for $\alpha>\bar{\alpha}_{1}$, that means if there exists a non-critical homoclinic orbit to the fixed point $\mathcal{O}_{\sigma^{1}}$, as explained in $\S 2.4$.

${ }^{10}$ For the cascade formed by the orbits $\mathcal{O}_{\varrho\left(\sigma_{1}\right)^{n}}$ with an odd $n$ this interval is relevant too, as it contains the point $f_{\mathcal{R}}\left(f_{\varrho}\left(x_{0}^{\varrho}\right)\right)$.
} 
the trajectories of the discontinuous flat top tent map converge to the Milnor attractor given by the unstable orbit $\mathcal{O}_{\sigma}$.

\subsection{Stable and unstable sets of the fixed point $\mathcal{O}_{\sigma^{1}}$}

It is worth to note also the following peculiarity of the discontinuous flat top tent map. As one can easily see, the unstable set of the unstable fixed point $\mathcal{O}_{\sigma^{1}}$ contains at least the interval

$$
I=\left[f_{\mathcal{R}}^{2}\left(\frac{1+\gamma}{2}\right), f_{\mathcal{R}}\left(\frac{1+\gamma}{2}\right)\right]
$$

independently on the value of $\beta$. Additionally, note that the interval $I$ intersects the middle partition $I_{\mathcal{C}}$, and therefore the unstable set of $\mathcal{O}_{\sigma^{1}}$ depends on the value $\beta$ on this interval. Clearly, any orbit started close to the unstable fixed point $\mathcal{O}_{\sigma^{1}}$ will reach the middle partition, and then if $\beta \in I$ it will remain inside $I$. Hence, for $\beta \in I$, the unstable set $W^{u}\left(\mathcal{O}_{\sigma^{1}}\right)$ is identical with $I$, as shown in Fig. $13 a$. Otherwise ${ }^{11}$, it contains also the forward orbit of $I_{\mathcal{C}}$, which is of course a set of points:

$$
W^{u}\left(\mathcal{O}_{\sigma^{1}}\right)=\left\{\begin{array}{lll}
I & \text { if } & \beta \in I \\
I \cup \bigcup_{n=0}^{\infty} f^{n}(\beta) & \text { if } & \beta \notin I
\end{array}\right.
$$

An example for this situation is shown in Fig. 13b. At the parameter values used in this figure, the attractor of the discontinuous flat top tent map is given by the orbit $\mathcal{O}_{\mathcal{C R} \mathcal{L}^{3}}$, which becomes therefore included into the the unstable set $W^{u}\left(\mathcal{O}_{\sigma^{1}}\right)$.

The situation with the stable set $W^{s}\left(\mathcal{O}_{\sigma^{1}}\right)$ of the unstable fixed point $\mathcal{O}_{\sigma^{1}}$ is also dependent on $\beta$, but in a different manner. If $\mathcal{O}_{\sigma^{1}}$ does not represent a Milnor attractor, that means $\beta \notin B_{\sigma^{1}}$, then $W^{s}\left(\mathcal{O}_{\sigma^{1}}\right)$ is only a set of points, which are mapped onto $\mathcal{O}_{\sigma^{1}}$ after some number of iteration steps (as it is usual for stable sets of unstable orbits). By contrast to this, for $\beta \in B_{\sigma^{1}}$ it is not difficult to see that the stable set $W^{s}\left(\mathcal{O}_{\sigma^{1}}\right)$ is no longer a set of points but a mixed set containing points and intervals. Indeed, calculating the preimages of $\mathcal{O}_{\sigma^{1}}$ (in other words, staring with $\mathcal{O}_{\sigma^{1}}$ and going backward) one reaches after some number of steps the middle partition $I_{C}$ and after that all the preimages of $I_{C}$ must be considered. Therefore, if $\beta \in B_{\sigma^{1}}^{\varrho}$ then the stable set $W^{s}\left(\mathcal{O}_{\sigma^{1}}\right)$ can be written as

$$
W^{s}\left(\mathcal{O}_{\sigma^{1}}\right)=\underbrace{\left(\bigcup_{i=0}^{m} f^{i}(\beta)\right)}_{(P)} \cup \underbrace{\left(\bigcup_{i=0}^{\infty} f^{-i}\left(I_{\mathcal{C}}\right)\right)}_{(I)} \text { with } m=|\varrho| .
$$

Hereby the set $(P)$ is a set of points, whereas the set $(I)$ consist of intervals (preimages of the middle partition $\left.I_{\mathcal{C}}\right)$. Moreover, note that in general the remaining set $[0,1] \backslash W^{s}\left(\mathcal{O}_{\sigma^{1}}\right)$ can contain other unstable orbits of the discontinuous flat top tent map and their preimages, but since close to $\alpha_{1}$ no other unstable orbits are possible, we conclude that in this case we have simply $W^{s}\left(\mathcal{O}_{\sigma^{1}}\right)=(0,1)$. Hence, for $\beta \in B_{\sigma^{1}}$ we have a non-empty intersection

$$
W^{u}\left(\mathcal{O}_{\sigma^{1}}\right) \cap W^{s}\left(\mathcal{O}_{\sigma^{1}}\right)=W^{u}\left(\mathcal{O}_{\sigma^{1}}\right) \cap(0,1)=W^{u}\left(\mathcal{O}_{\sigma^{1}}\right) \neq \emptyset
$$

and therefore there exists a homoclinic orbit to the the unstable fixed point $\mathcal{O}_{\sigma^{1}}$.

At this point it is worth to note the one more property of the discontinuous flat top tent map. Recall that the presence of a homoclinic orbit to an unstable fixed point (or an unstable cycle) is typically associated with the

\footnotetext{
${ }^{11}$ Of course, Eq. (37) can also be written simply as $W^{u}\left(\mathcal{O}_{\sigma^{1}}\right)=I \cup \bigcup_{n=0}^{\infty} f^{n}(\beta)$, since in the case $\beta \in I$ the forward orbit of $I_{\mathcal{C}}$ is a subset of $I$. Nevertheless, we prefer to distinguish between the cases $\beta \in I$ and $\beta \notin I$, because in the first case the unstable set of an unstable fixed point is given simply by an interval (as it is usually the case), whereas in the second case it represents a mixed set containing an interval and a set of points. This last situation represents a peculiarity of maps with a horizontal part.
} 

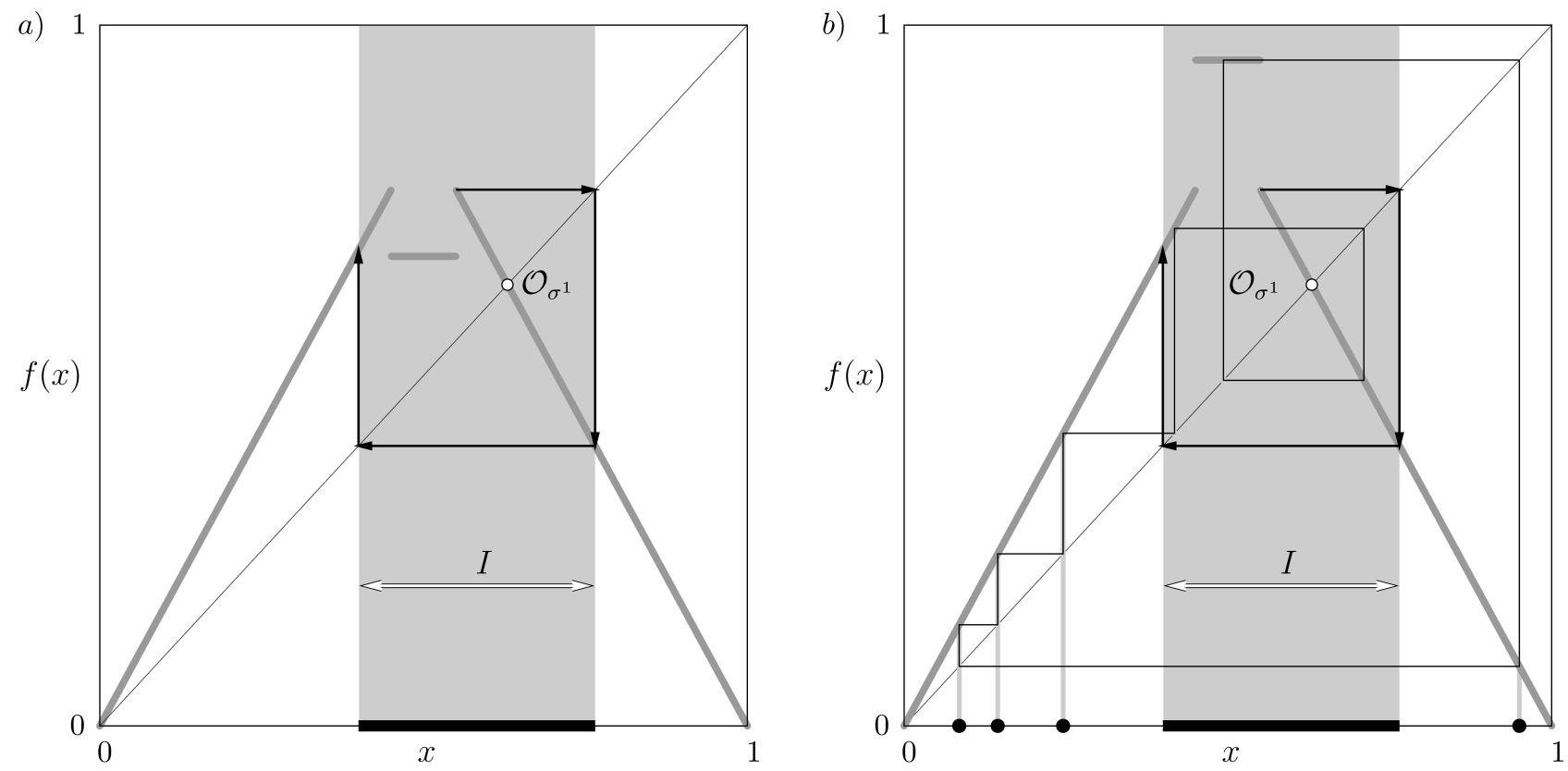

Figure 13. Unstable set $W^{u}\left(\mathcal{O}_{\sigma^{1}}\right)$ of the unstable fixed point $\mathcal{O}_{\sigma^{1}}$ for $a=0.85$ (shown black on the horizontal axis). In a) $\beta=0.67 \in I$, therefore $W^{u}\left(\mathcal{O}_{\sigma^{1}}\right)$ is given by the interval $I$ only. In (b) $\beta=0.95 \notin I$, therefore $W^{u}\left(\mathcal{O}_{\sigma^{1}}\right)$ contains additionally four singular points outside of the interval $I$.

presence of an infinite number of further unstable orbits and chaos (either a chaotic attractor or at least a chaotic repeller). However, as shown in [6], this is true only if the homoclinic orbit is non-critical (structural stable). It is also shown in the cited work, that in the case of a critical (structural unstable) homoclinic orbit both situations are possible: chaos may exist or not exist. As one can see, the homoclinic orbits of the discontinuous flat top tent map containing a point in the middle partition (see Fig. $14 b$ for an example) are critical, since any perturbation of $\beta$ leads the homoclinic orbit to disappear. In this case, neither a chaotic attractor nor a chaotic repeller is possible, as the unstable fixed point $\mathcal{O}_{\sigma^{1}}$ is a Milnor attractor and its basin covers the complete interval ] $0,1[$.

For increasing values of $\alpha$ also homoclinic orbits to the unstable fixed point $\mathcal{O}_{\sigma^{1}}$ appear, containing points on the left and on the right partitions only (and consequently representing homoclinic orbits also for the tent map). For the first time this happens at the parameter value $\bar{\alpha}$ defined by the condition

$$
f_{\mathcal{L}}\left(f_{\mathcal{R}}\left(f_{\mathcal{R}}\left(\frac{1+\gamma}{2}\right)\right)\right)=x_{\sigma^{1}}
$$

Fig. $14 a$ shows a homoclinic orbit to the unstable fixed point $\mathcal{O}_{\sigma^{1}}$ close to this value. At the parameter value $\alpha=\bar{\alpha}$ the homoclinic orbit is critical (indeed, for decreasing $\alpha$ the orbit disappears), but associated with the presence of a chaotic repeller, as we will see in $\S 2.5$. For $\alpha>\bar{\alpha}$ this homoclinic orbits is non-critical and therefore the chaotic repeller exists for any $\alpha>\bar{\alpha}$ and for any $\beta$ (since the value of $\beta$ plays no role for the orbits which do not contain any points on the middle partition).

\subsection{Period-2 orbit $\mathcal{O}_{\sigma^{2}}$ as a Milnor attractor and the further cascade up to $\alpha_{\infty}$}

The situation described above close to $\alpha_{1}$ persists until the unstable period-2 orbit $\mathcal{O}_{\sigma^{2}}=\left\{x_{\sigma^{2}}^{0}, x_{\sigma^{2}}^{1}\right\}$ appears (becomes non-virtual). This occurs at the parameter value $\alpha_{2}$ which is defined by the condition $f_{\mathcal{R}}\left(f_{\mathcal{L}}((1-\right.$ 
a)

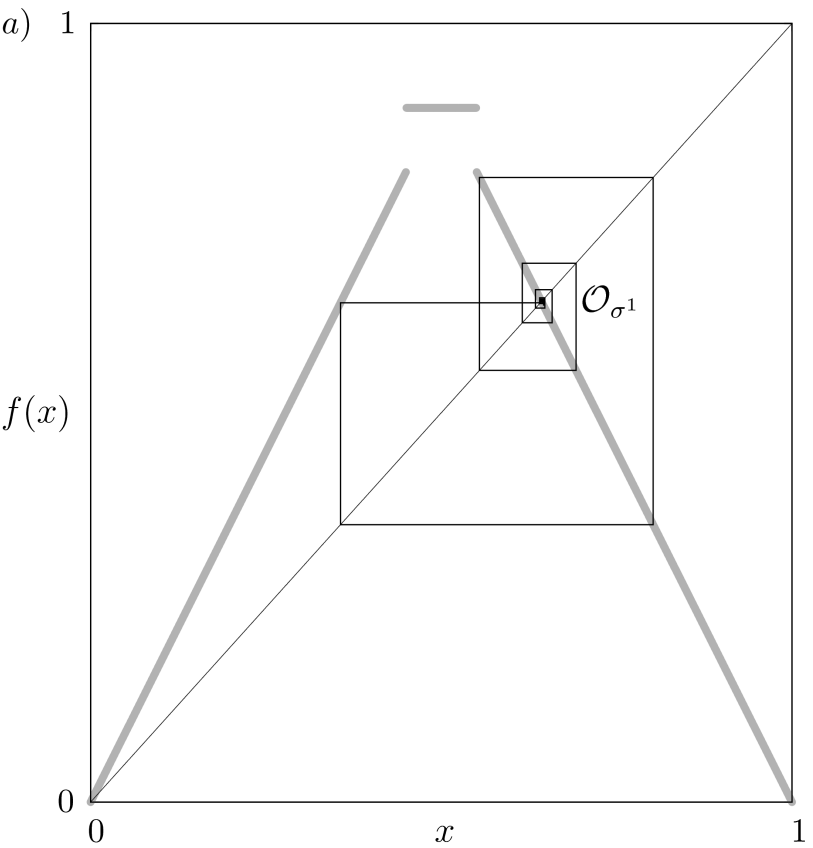

b)

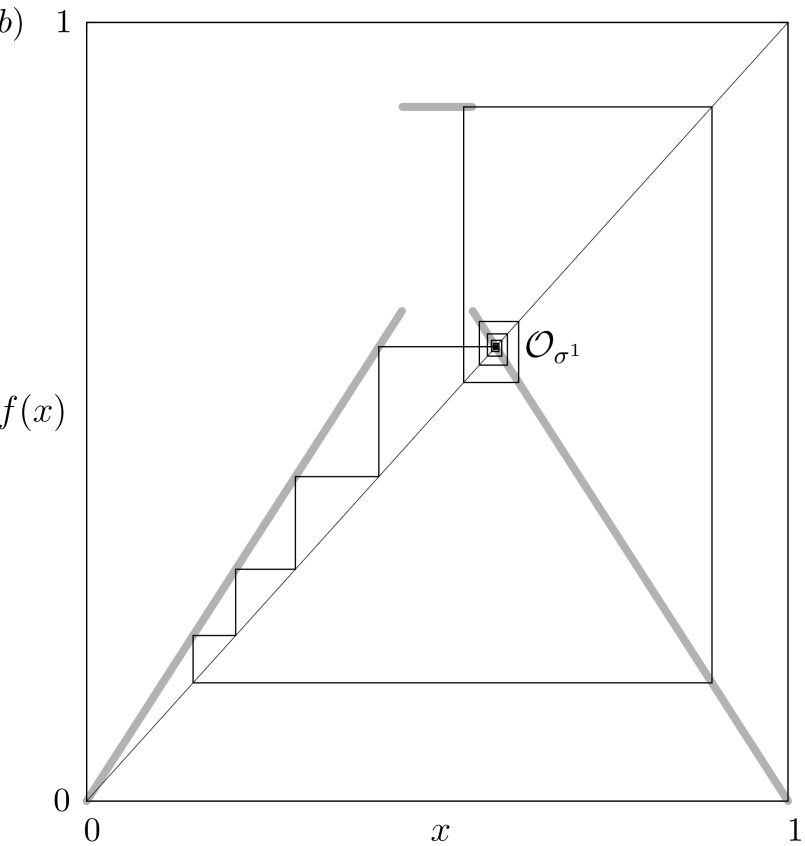

FiguRE 14. Homoclinic orbits to the unstable fixed point $\mathcal{O}_{\sigma^{1}}$. In (a), the homoclinic orbit is located on the partitions $I_{\mathcal{L}}$ and $I_{\mathcal{R}}$ only, the orbit is non-critical, and therefore the situation is as usual (the unstable fixed point $\mathcal{O}_{\sigma^{1}}$ represents a snap-back repeller, and the presence of the homoclinic orbit is associated with chaos). In (b) the homoclinic orbit is critical (as it contains a point located on the middle partition $I_{\mathcal{C}}$ ), and therefore the situation is different (the unstable fixed point $\mathcal{O}_{\sigma^{1}}$ represents a Milnor attractor, and the presence of the homoclinic orbit is not associated with chaos). Parameters: $a=0.9$ (a), $a=0.7$ (b), $\beta \approx 0.89152$.

$\gamma) / 2))=(1-\gamma) / 2$ and given by

$$
\alpha_{2}=\frac{1+\sqrt{2 \gamma-\gamma^{2}}}{2(1-\gamma)}
$$

At this point the complexity of the bifurcation structure increases again. The set $L_{2}$ of the symbolic sequences of the orbits emerging at the point $\alpha_{2}$ can be written as

$$
L_{2}=\left\{\varrho\left(\sigma^{2}\right)^{n} \mid \varrho \in L_{1}, n \geq 0\right\} .
$$

An example for the situation close to $\alpha=\alpha_{2}$ is shown in Fig. 15. In the presented region, for $\alpha<\alpha_{2}$ only the orbits $\mathcal{O}_{\mathcal{C R} \mathcal{L}^{4} \mathcal{R}}$ and $\mathcal{O}_{\mathcal{C R} \mathcal{L}^{4} \mathcal{R}^{3}}$ exist and their existence regions are adjoining. For $\alpha>\alpha_{2}$, that means when the unstable period-2 orbit $\mathcal{O}_{\sigma^{2}}$ exists, between these existence regions two period incrementing cascade

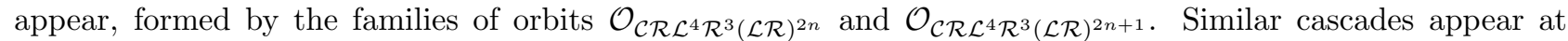
$\alpha=\alpha_{2}$ between each pair of periodicity regions which are adjoining for $\alpha<\alpha_{2}$, so that the situation remains qualitatively the same as shown schematically in Fig 10, with $\sigma=\sigma^{2}$ and $\tilde{\alpha}=\alpha_{2}$. At the parameter values where the period incrementing cascades converge to, the unstable orbit $\mathcal{O}_{\sigma^{2}}$ becomes a Milnor attractor in the same way as it was described above for the unstable fixed point $\mathcal{O}_{\sigma^{1}}$. The parameter values where $\mathcal{O}_{\sigma^{2}}$ represents a Milnor attractor are defined by the condition that the value of $\beta$ is set to one of the points of this orbit or to some of their preimages:

$$
B_{\sigma^{2}}(\alpha):=\left\{\beta \mid \exists k \geq 0: f^{k}(\beta) \in\left\{x_{\sigma^{2}}^{0}, x_{\sigma^{2}}^{1}\right\}\right\}
$$




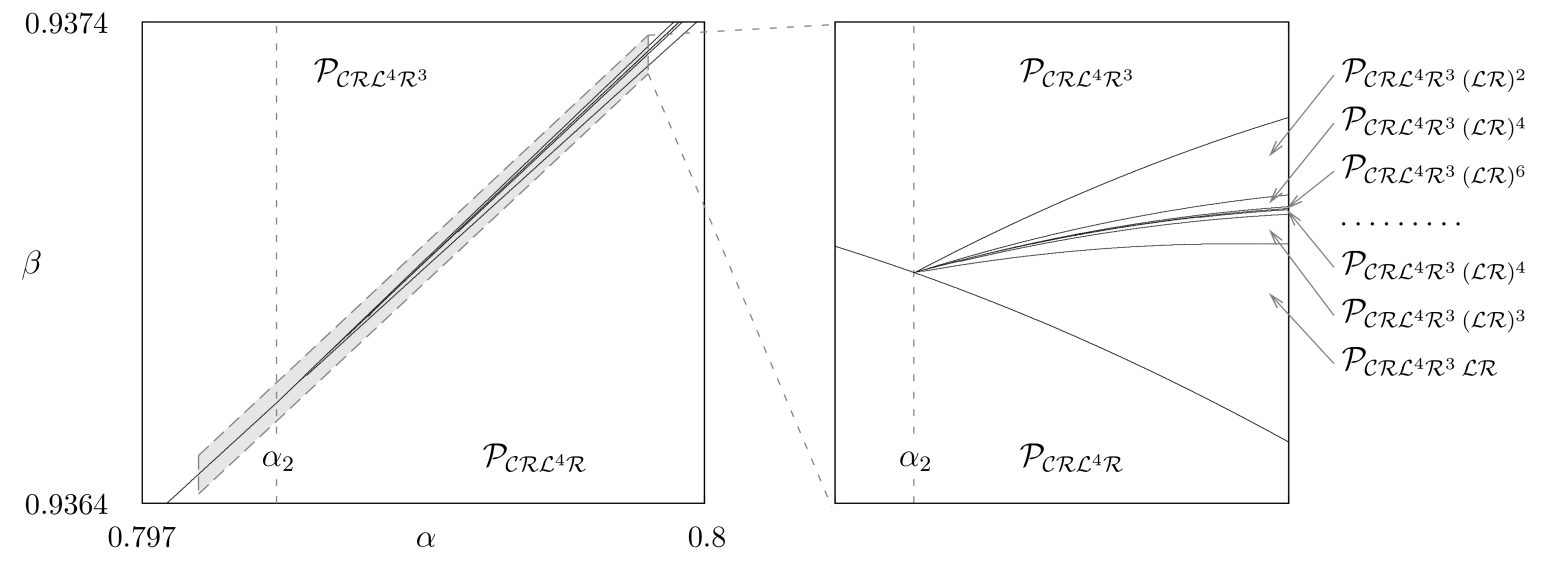

Figure 15. A small region in the $(\alpha, \beta)$ parameter plane is shown close to the bifurcation line $\alpha=\alpha_{2}$ where the unstable period- 2 orbit $\mathcal{O}_{\sigma^{2}}$ emerges. The quadrilateral region (parallelogram) marked with dashed lines is shown enlarged.

Note that the set $B_{\sigma^{2}}(\alpha)$ contains all accumulation points of the nested period incrementing cascades which are shown in Fig. $2 c$ except for those which were already present in Fig. 2b. Indeed, as one can see in Fig. 3, the parameter value $\alpha=0.85$ used in Fig. $2 c$ is above the value $\alpha_{2}$ but still below the value $\alpha_{3}$ where the next unstable orbit with period 4 appears.

Similarly to the previous case we can also define the sets of parameters for which the path from the middle partition to the Milnor attractor given by the unstable orbit $\mathcal{O}_{\sigma^{2}}$ is of length $k$

$$
\begin{aligned}
B_{\sigma^{2}}^{k}(\alpha) & :=B_{\sigma^{2}}^{k, 0}(\alpha) \cup B_{\sigma^{2}}^{k, 1}(\alpha) \text { with } \\
B_{\sigma^{2}}^{k, n}(\alpha) & :=\left\{\beta \mid f^{k}(\beta)=x_{\sigma^{2}}^{n}, \text { and } \nexists j<k: f^{j}(\beta)=x_{\sigma^{2}}^{n}, n=0,1\right\} .
\end{aligned}
$$

As an example, in Fig. 16 the sets $B_{\sigma^{2}}^{k}$ for $k \leq 7$ are shown. As one can see, some of the curves belonging to these sets emerge at the value $\alpha=\alpha_{2}$, whereas the other values emerge for larger values of $\alpha$.

There exist also some minor differences to the previous case, but they are not significant. One difference is that the unstable orbit $\mathcal{O}_{\sigma^{2}}$ is not the only unstable object existing for $\alpha>\alpha_{2}$, and hence its stable set $W^{s}\left(\mathcal{O}_{\sigma^{2}}\right)$ is not the complete interval $] 0,1[$ but the set $] 0,1\left[\backslash W^{s}\left(\mathcal{O}_{\sigma^{1}}\right)\right.$. However, for a given value of $\alpha$ the same value of $\beta$ can not belong to both $B_{\sigma^{1}}(\alpha)$ and $B_{\sigma^{2}}(\alpha)$, or in other words $\beta \in B_{\sigma^{2}}(\alpha)$ implies $\beta \notin B_{\sigma^{1}}(\alpha)$. Therefore, for $\beta \in B_{\sigma^{2}}(\alpha)$ the set $W^{s}\left(\mathcal{O}_{\sigma^{1}}\right)$ consists of points and hence the stable set $W^{s}\left(\mathcal{O}_{\sigma^{2}}\right)$ covers the interval ]0, $1[$ up to a set of the zero Lebesgue measure. As a consequence, for $\beta \in B_{\sigma^{2}}(\alpha)$ and $\alpha$ close to $\alpha_{2}$ there exist critical homoclinic orbits to the period-2 orbit $\mathcal{O}_{\sigma^{2}}$, similarly to the previous case not associated with chaos.

For increasing $\alpha$, the unstable orbits $\mathcal{O}_{\sigma^{k}}$ with periods $2^{k}$ with $k=0,1,2,3 \ldots$ appear one after the other at the parameter values $\alpha_{1}<\alpha_{2}<\alpha_{3}<\alpha_{4}<\ldots$ At the $i$ th bifurcation in this sequence a family of orbits

$$
L_{i}=\left\{\varrho\left(\sigma^{i}\right)^{n} \mid \varrho \in L_{i-1}, n \geq 0\right\}
$$

emerges, so that the overall set of orbits existing after the bifurcation is given by $\bigcup_{k=0}^{i} L_{k}$. For each $\varrho \in L_{i-1}$ the incrementing cascade formed by orbits with the symbolic sequences $\varrho\left(\sigma^{i}\right)^{n}$ converges to the parameter value where the orbit with the symbolic sequence $\sigma^{i}$ represents a Milnor attractor.

The sequence of bifurcation points $\alpha_{i}$, with $i=1,2,3 \ldots$ accumulates at the so-called Feigenbaum point $\alpha_{\infty}$. An approximation for this value can be found numerically by calculating values $\alpha_{i}$ up to some number $k$ which do not need to be large. In fact, we found out that already for $k=7$ the difference $\alpha_{7}-\alpha_{6}$ is less than $10^{-15}$, so 


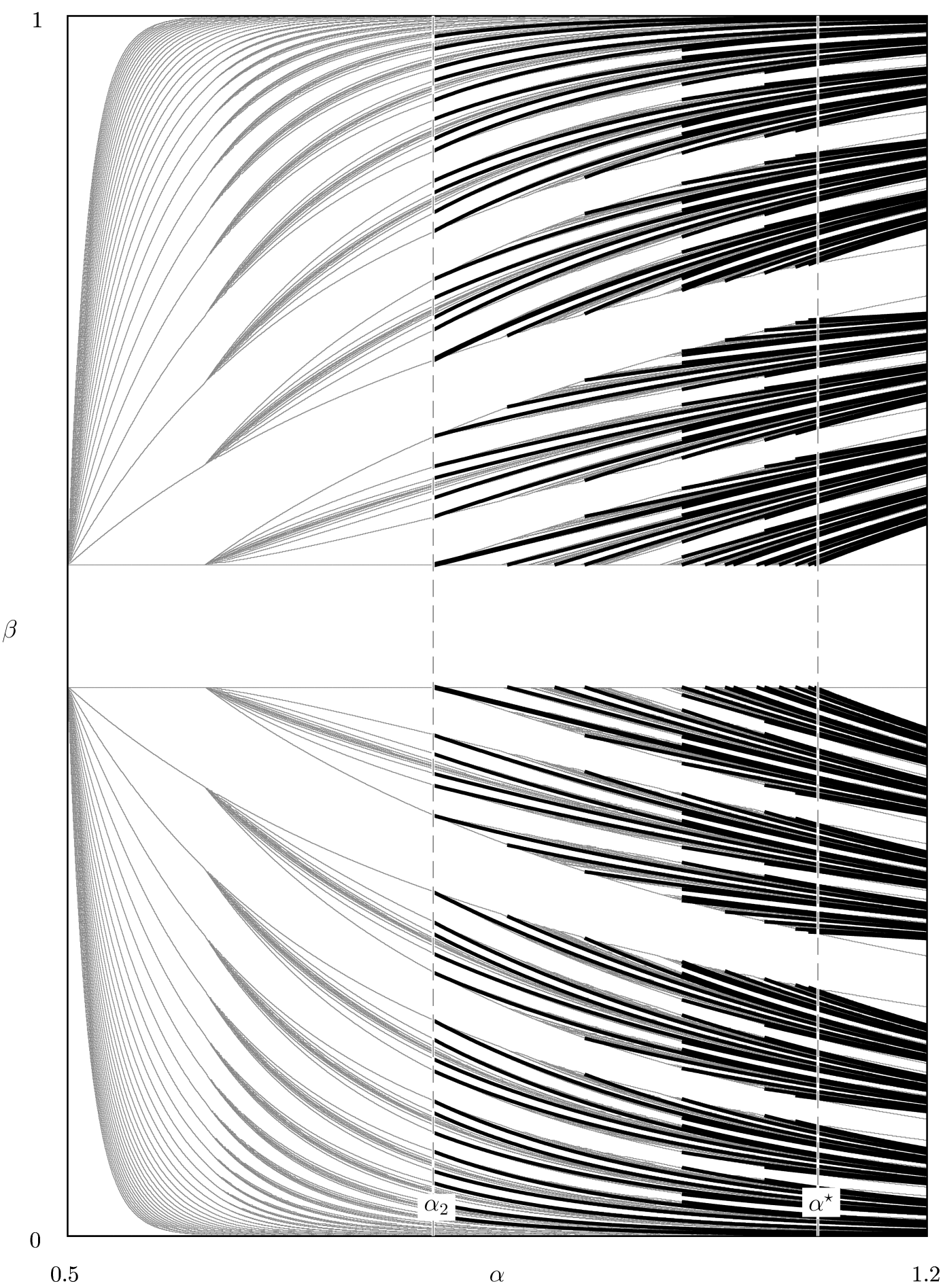

Figure 16. Black curves shows the sets $B_{\sigma^{2}}^{k}$ with $k \leq 7$ of the parameter values where the unstable period- $2 \mathcal{O}_{\sigma^{2}}$ represents a Milnor attractor. 
that we can consider the value $\alpha_{7} \approx 0.85733979614229$ as a good approximation for $\alpha_{\infty}$. Similar to the logistic map, for $\alpha>\alpha_{\infty}$ there exists a chaotic repeller, so that at the bifurcation points $\bar{\alpha}_{i+1}<\bar{\alpha}_{i}<\cdots<\bar{\alpha}_{1}$ the corresponding orbits $\mathcal{O}_{\sigma^{i+1}}, \mathcal{O}_{\sigma^{i}}, \ldots, \mathcal{O}_{\sigma^{1}}$ are critical homoclinic and associated with chaos.

Combining Eqs. (24), (33), (42), (45), we can state as a final result that the set of all stable periodic orbits existing for $\alpha_{m}<\alpha \leq \alpha_{m+1}$ correspond to the following set of symbolic sequences:

$$
\left\{\mathcal{C} \mathcal{R}^{k} \mathcal{L}^{n_{0}}\left(\sigma^{1}\right)^{n_{1}}\left(\sigma^{2}\right)^{n_{2}} \ldots\left(\sigma^{m}\right)^{n_{m}} \mid k \in\{0,1\}, n_{i} \geq 0, i=0, \ldots, m\right\}
$$

Hence, for $m \rightarrow \infty$ we obtain the set of all stable periodic orbits which emerge for $\alpha<\alpha_{\infty}$. Of course, all these orbits persist for $\alpha \geq \alpha_{\infty}$.

\subsection{Fixed point $\mathcal{O}_{\mathcal{L}}$ as a Milnor attractor}

It is remarkable that in Eq. (46) the first letter $\mathcal{R}$ requires a special treatment. Indeed, this letter can appear zero or one times (in other words, it can appear once or not appear at all) whereas all other subsequences $\left(\mathcal{L}, \sigma^{1}, \ldots, \sigma^{m}\right)$ can appear an arbitrary number of times. To explain that, let us turn back to the situation for $1 / 2<\alpha<\alpha_{1}$ discussed before. It turns out that the two cascades existing in these parameter range can easily be explained in the same manner as we explained the situation in the complete cascade occurring for $\alpha_{1}<\alpha<\alpha_{\infty}$. Recall that the unstable fixed point $\mathcal{O}_{\mathcal{R}} \equiv \mathcal{O}_{\sigma_{1}}$ we discussed in detail before is not the only fixed point of the discontinuous flat top tent map. By contrast, there exists also the fixed point $\mathcal{O}_{\mathcal{L}}$ which is stable for $\alpha<1 / 2$ and unstable for $\alpha>1 / 2$. Then, by using a suitable value of $\beta$, this unstable fixed point may be made to a Milnor attractor. The only difference to the other fixed point regards the number of preimages. It is easy to see that for any $1 / 2<\alpha<\alpha^{\star}$ the fixed point $\mathcal{O}_{\mathcal{L}}$ has only one rank-1 preimage different from itself and no preimages of any higher ranks:

$$
B_{\mathcal{L}}^{0}(\alpha)=B_{\mathcal{L}}^{\varepsilon}(\alpha)=\{0\}, \quad B_{\mathcal{L}}^{1}(\alpha)=B_{\mathcal{L}}^{\mathcal{R}}(\alpha)=\{1\}, \quad \forall k>1: \quad B_{\mathcal{L}}^{k}(\alpha)=\emptyset
$$

Therefore, for any $1 / 2<\alpha<\alpha^{\star}$ the fixed point $\mathcal{O}_{\mathcal{L}}$ represents a Milnor attractor for $\beta=0$ and $\beta=1$. Moreover, the set $L_{0}$ of symbolic sequences of the stable orbits which emerge at $\alpha=1 / 2$ (see Eq. (24)) can be written again as $\left\{\mathcal{C} \varrho(\sigma)^{n} \mid n \geq 0\right\}$ where $\sigma=\mathcal{L}$ is the symbolic sequence of the Milnor attractor $\mathcal{O}_{\mathcal{L}}$ and $\varrho \in\{\varepsilon, \mathcal{R}\}$ for $\alpha<\alpha^{\star}$ are the symbolic sequences of the two possible orbits leading from $I_{\mathcal{C}}$ onto this Milnor attractor.

At $\alpha=\alpha^{\star}$, the discontinuous flat top tent map becomes surjective onto [0,1], which gives rise to an infinitude of preimages of the point $x=1$ corresponding to the aforementioned rank-1 preimage of the Milnor attractor $\mathcal{O}_{\mathcal{L}}$. Since every preimage of $x=1$ has both $\mathcal{L}$ - and $\mathcal{R}$-preimages, for all $k$ every symbolic sequence $\varrho=\varrho_{0} \varrho_{1} \ldots \varrho_{k 1}$ which ends with $\mathcal{R}$ represents a possible path from the middle partition to the unstable fixed point $\mathcal{O}_{\mathcal{L}}$ and the corresponding set of values of $\beta$ at which $\mathcal{O}_{\mathcal{L}}$ becomes a Milnor attractor is given by

$$
B_{\mathcal{L}}^{k}\left(\alpha^{\star}\right)=\left\{B_{\sigma^{1}}^{\varrho}\left(\alpha^{\star}\right) \mid \varrho_{i} \in\{\mathcal{L}, \mathcal{R}\}, i=0 . . k-2, \varrho_{k-1}=\mathcal{R}\right\}
$$

\section{Bifurcation Structure For $\alpha>\alpha_{\infty}$}

After the Feigenbaum point $\alpha=\alpha_{\infty}$ the situation becomes more rich. To explain that, let us consider again the logistic map. For this map it is known that the stable periodic orbits are organized according the so-called universal sequence (U-sequence for short) introduced by Metropolis, Stein and Stein in [12]. Independently, this structure was also described by Gumowski an Mira in [9] where it was called the box-within-a-box structure. Note that the U-sequence can not be written in a closed form but results from an algorithm which allows to determine the symbolic sequences ("patterns") for all super-stable periodic orbits up to a given period existing between the existence regions of two given super-stable periodic orbits (which will be assumed to be a priori known). It is also known for the tent map that the unstable periodic orbits in this system appear in the same order except that the period doubling cascades in this case are compressed to one point (see for an example 
the first doubling cascade mentioned in $\S 2.1$ ). However, in the case of the discontinuous flat top tent map the cascades are expanded again (see the explanation in $\S 2.1$ ). Therefore, the order of the appearance of unstable periodic orbits in the discontinuous flat top tent map is the same as the order of the appearance of stable periodic orbits in the logistic map. Recall also, that any unstable periodic orbit, once appeared at some value $\hat{\alpha}$, persists then for any larger value $\alpha>\hat{\alpha}$. Hence, the crucial difference between the situations before and after $\alpha_{\infty}$ is that for any $\alpha<\alpha_{\infty}$ there exists only a finite number of unstable periodic orbits, whereas for $\alpha \geq \alpha_{\infty}$ this number is infinite. Moreover, for $\alpha>\alpha_{\infty}$ the symbolic sequences of these orbits can not be written in a closed form. By contrast, the only way to specify them explicitly is to fix some value for the maximal period and to generate all the sequences up to this period using the rules given by the U-sequence. Therefore, it seems to be not possible to specify all stable orbits existing at some particular value $\alpha=\hat{\alpha}>\alpha_{\infty}$. Instead, for a given value $\alpha_{\infty}<\hat{\alpha}<\alpha^{\star}$ one can in principle proceed as follows.

(1) Fix some maximal period $\bar{p}$. It is worth to note that this period will be not the maximal period of the stable periodic orbits of the discontinuous flat top tent map we will determine, but an auxiliary value we need for the calculations.

(2) Using the known rules describing the U-sequence determine all unstable periodic orbits of the tent map with the periods not higher than $\bar{p}$ existing for $\alpha<\hat{\alpha}$.

(3) Starting with the set of stable periodic orbits defined by Eq. (46) for $m \rightarrow \infty$ which exists at the value $\alpha=\alpha_{\infty}$ one can proceed for $\alpha$ increasing from $\alpha_{\infty}$ to $\hat{\alpha}$ and extend the set of stable periodic orbits at each

point where an unstable periodic orbit emerges in the same way as given by Eq. (45).

As one can see, this procedure is too complicated for a practical application, although theoretically correct. Therefore, the algorithmic approach for determining the symbolic description of the stable periodic orbits existing at a given value of $\hat{\alpha}$ represents the only feasible solution for $\alpha_{\infty}<\hat{\alpha}<\alpha^{\star}$ known so far.

A detailed investigation of the domain $\alpha_{\infty}<\alpha<\alpha^{\star}$ is beyond the scope of this work. In this parameter interval not only new unstable periodic orbits emerge, organized according to the U-sequence, and causing new period incrementing cascades formed by stable periodic orbits to occur, but also the unstable periodic orbits already existing for smaller values of $\alpha$ undergo infinite cascades of homoclinic bifurcations. For each unstable periodic orbit, these bifurcations occur at the parameter value where the points of this orbit get new preimages and therefore new homoclinic orbits emerge. After the homoclinic bifurcations these homoclinic orbits are noncritical, which implies the existence of a chaotic repeller (as chaotic attractors can not exist in the discontinuous flat top tent map). The presence of new preimages implies also new paths from the middle partition to the already existing unstable orbits, so that the set of the curves in the parameter space where this orbit represents a Milnor attractor will be extended. This can clearly be seen for the unstable fixed point $\mathcal{O}_{\sigma^{1}}$ and the unstable period-2 orbit $\mathcal{O}_{\sigma^{2}}$ in Figs.16 and 9, respectively. However, it is evident that the basic organizing principles of the domain $\alpha>\alpha_{\infty}$ can be described combining the well-known properties of the usual tent map with the properties of the discontinuous flat top tent map described for $\alpha<\alpha_{\infty}$.

\section{Conclusions}

In the presented work we investigated the discontinuous flat top tent map and demonstrated that this system has several properties which are quite unusual and were not observed before when dealing with other dynamical systems. Of course, the map we investigated may look quite particular and hence the question arises whether some of our results may be useful in a more general context. In fact, the discontinuous flat top tent map belongs to two classes of piecewise-smooth maps which were barely investigated until now, namely to the class of maps with a constant value on some interval of their definition (denoted as maps with a horizontal part throughout this work) and to the class of discontinuous maps defined on more than two partitions. Therefore it is evident that some of the phenomena we reported for the discontinuous flat top tent map can have a general meaning for these classes of maps.

We reported the following peculiarities of the discontinuous flat top tent map: 
- Both the stable and the unstable sets of unstable orbits may represent "mixed" sets containing points and intervals. This is by contrast to the maps without a constant interval, where the stable sets of unstable orbits represent sets of points and the unstable sets represent sets of intervals only.

- The unstable orbit of the discontinuous flat top tent map may become a Milnor attractor.

As these properties are due to the constant value in an interval of the map definition, it is evident that they are general for maps which such a constant value. Of course, in other maps, where the value in the constant interval can not be varied independently of other parameters, they are not necessarily fulfilled for all unstable orbits.

Furthermore, we demonstrated the following property of the discontinuous flat top tent map:

- There exists a strong connection between the existence of stable and unstable orbits. Such kind of connection is basically not unusual, as for instance in smooth systems a stable and an unstable orbit may appear together via a saddle-node bifurcation. However, in the discontinuous flat top tent map the situation is more rich, and the appearance of a single unstable orbit lead an infinite number of stable periodic orbits to appear. Moreover, these stable orbits are organized in an infinite number of period incrementing cascades, originating from an infinite number of codimension-2 bifurcation points (codimension-2 big bang bifurcation points). These cascades converge to the parameter values where the only attractor of the discontinuous flat top tent map is given by the unstable orbit mentioned above, which represents therefore a Milnor attractor.

It is still an open question for which class of maps a similar result can be obtained. It can be expected, that not every map with a constant value on some interval will demonstrate that, since the signs of the slopes on the left and the right partitions may be significant here. However, a convergence of the bifurcation cascades to a value where a Milnor attractor exists may be a quite general phenomenon for maps with a constant value on some interval.

Regarding discontinuous maps defined on more than two partitions, the following should be mentioned. When dealing with piecewise-smooth maps defined on two partitions and assuming the system function to be monotonous at each of the partitions, it is known that the bifurcation structures are different depending on the fact whether the system function on the left and on the right partitions is increasing or decreasing. Correspondingly, for maps defined on two partitions (that means, with one discontinuity) there are three possible cases (the system function is increasing on both partitions, increasing on one partition and decreasing on the other one, and decreasing on both partitions)

In the case of maps defined on three partitions, there are much more possible cases, and the only one of them, for which some preliminary results are already known, is the case that the system function is increasing on all three partitions [17]. By contrast, for the cases that the shape of the system function is according to the configuration increasing - increasing - decreasing and increasing - decreasing - decreasing, no results are available so far. However, the results we reported in this work for the discontinuous flat top tent map represent in some sense a switching case (increasing - constant - decreasing) between these two configurations. Therefore one can conclude that at least the basic organization principles of the robust orbits must be present also in the two configurations mentioned above.

\section{REFERENCES}

[1] K. T. Alligood, T. D. Sauer, and J. A. Yorke. Chaos: An Introduction to Dynamical Systems. Springer-Verlag, Berlin, 1996.

[2] V. Avrutin, B. Futter, and M. Schanz. The discontinuous flat top tent map and the nested period incrementing bifurcation structure. (submitted for publication), 2011.

[3] V. Avrutin, M. Schanz, and L. Gardini. Self-similarity of the bandcount adding structures: calculation by map replacement. Regular and Chaotic Dynamics, 15(6):685-703, 2010.

[4] V. Avrutin, M. Schanz, and B. Schenke. On a bifurcation structure mimicking period adding. Proc. R. Soc. A, 467(2129):1503$1518,2011$.

[5] N.J. Corron, S.D. Pethel, and B.A. Hopper. Controlling Chaos with Simple Limiters. Phys. Rev. Lett, 84(17):3835, 2000.

[6] L. Gardini, I. Sushko, V. Avrutin, and M. Schanz. Critical homoclinic orbits lead to snap-back repellers. Chaos, Solitons 8 Fractals, 44:433-449, 2011.

[7] A. Garfinkel, M. Spano, W. Ditto, and J. Weiss. Controlling cardiac chaos. Science, 257:1230, 1992. 
[8] L. Glass and W. Zeng. Bifurcations in Flat-Topped Maps and the Control of Cardiac Chaos. International Journal of Bifurcation and Chaos, 4(4):1061-1067, 1994.

[9] I. Gumowski and C. Mira. Accumulation de bifurcations dans une récurrence. Comptes Rendus Acad. Sc. Paris, Série A, pages 45-48, 1975.

[10] T. Kabe, S. Parui, H. Torikai, S. Banerjee, and T. Saito. Analysis of Piecewise Constant Models of Current Mode Controlled DC-DC Converters. IEICI Trans. Fundamentals, E90-A(2):448, 2007.

[11] Y. Matsuoka and T. Saito. Rotation Map with a Controlling Segment and Its Application to A/D Converters. IEICI Trans. Fundamentals, E91-A(7):1725-1732, 2008.

[12] N. Metropolis, M. L. Stein, and P. R. Stein. On Finite Limit Sets for Transformations on the Unit Interval. J. Comb. Theory, A15:25-44, 1973.

[13] J. W. Milnor. On the Concept of Attractor. Commun. Math. Phys., 99:177-195, 1985.

[14] K. Myneni, T. A. Barr, N. J. Corron, and S. D. Pethel. New method for the control of fast chaotic oscillations. Phys. Rev. Lett., 83:2175, 1999.

[15] R. Stoop and C. Wagner. Scaling Properties of Simple Limiter Control. Phys. Rev. Lett, 90(15), 2003.

[16] I. Sushko and L. Gardini. Degenerate Bifurcations and Border Collisions in Piecewise Smooth 1D and 2D Maps. Int. J. Bifurcation Chaos, 20(7):2045-2070, 2010.

[17] F. Tramontana, L. Gardini, V. Avrutin, and M. Schanz. New Adding Phenomena in Piecewise Linear Maps with two discontinuities. Int. J. Bifurcat. Chaos, 2011. (accepted for publication).

[18] C. Wagner and R. Stoop. Optimized chaos control with simple limiters. Phys. Rev. E, 63:017201, 2000.

[19] C. Wagner and R. Stoop. Renormalization Approach to Optimal Limiter Control in 1-D Chaotic Systems. J. Stat. Phys., 106:97-107, 2002.

[20] K. Zyczkowski and E.M. Bollt. On the entropy devils staircase in a family of gap-tent maps. Physica D, 132:392-410, 1999. 